\title{
Atrial natriuretic peptide promotes cardiomyocyte survival by cGMP-dependent nuclear accumulation of zyxin and Akt
}

\author{
Takahiro Kato, ${ }^{1}$ John Muraski, ${ }^{1}$ Yan Chen,, ${ }^{1}$ Yasuyuki Tsujita, ${ }^{1}$ Jason Wall, ${ }^{1}$ \\ Christopher C. Glembotski, ${ }^{1}$ Erik Schaefer, ${ }^{2}$ Mary Beckerle, ${ }^{3}$ and Mark A. Sussman ${ }^{1}$ \\ ${ }^{1}$ San Diego State University Heart Institute, Department of Biology, San Diego, California, USA. Biosource International, Hopkinton, Massachusetts, USA. \\ 3Huntsman Cancer Institute, Departments of Biology and Oncological Sciences, University of Utah, Salt Lake City, Utah, USA.
}

\begin{abstract}
This study delineates a mechanism for antiapoptotic signaling initiated by atrial natriuretic peptide (ANP) stimulation leading to elevation of cGMP levels and subsequent nuclear accumulation of Akt kinase associated with zyxin, a cytoskeletal LIM-domain protein. Nuclear targeting of zyxin induces resistance to cell death coincident with nuclear accumulation of activated Akt. Nuclear translocation of zyxin triggered by cGMP also promotes nuclear Akt accumulation. Additional supportive evidence for nuclear accumulation of zyxin-enhancing cardiomyocyte survival includes the following: (a) promotion of zyxin nuclear localization by cardioprotective stimuli; (b) zyxin association with phospho- $\mathrm{Akt}^{473}$ induced by cardioprotective stimuli; and (c) recruitment of zyxin to the nucleus by activated nuclear-targeted Akt as well as recruitment of Akt by nuclear-targeted zyxin. Nuclear accumulation of zyxin requires both Akt activation and nuclear localization. Potentiation of cell survival is sensitive to stimulation intensity with high-level induction by ANP or cGMP signaling leading to apoptotic cell death rather than enhancing resistance to apoptotic stimuli. Myocardial nuclear accumulation of zyxin and Akt responds similarly in vivo following treatment of mice with ANP or cGMP. Thus, zyxin and activated Akt participate in a cGMP-dependent signaling cascade leading from ANP receptors to nuclear accumulation of both molecules. Nuclear accumulation of zyxin and activated Akt may represent a fundamental mechanism that facilitates nuclear-signal transduction and potentiates cell survival.
\end{abstract}

\section{Introduction}

Members of the natriuretic peptide family, atrial natriuretic peptide (ANP), brain natriuretic peptide, and $\mathrm{C}$ type natriuretic peptide, serve as physiological regulators of homeostasis including control of fluid retention and blood pressure. Clinical treatment of various cardiovascular pathologies shows that natriuretic peptides exert beneficial effects in arterial hypertension $(1,2)$, myocardial infarction (3), coronary artery disease (4-7), and chronic heart failure (8-13). Collectively, these results suggest that ANP is cardioprotective at a plasma concentration of approximately 3 $\mathrm{ng} / \mathrm{ml}\left(10^{-9} \mathrm{M}\right)$ in treated patients, in agreement with in vitro data using $10^{-9} \mathrm{M}$ ANP exposure to inhibit apoptotic cell death induced by serum deprivation in cultured nonmyocytes (14) as well as similar protective effects in other cells $(15,16)$. In comparison, relatively high ANP concentrations $\left(10^{-7} \mathrm{M}\right)$ promote apoptosis in various cell types (17-18). These data suggest that very different effects of ANP on preventing or inducing apoptosis may occur at therapeutic $\left(10^{-9} \mathrm{M}\right)$ or experimentally elevated $\left(10^{-6} \mathrm{M}\right)$ concentrations, respectively. Antiapoptotic effects of natriuretic peptide are mediated via activation of particulate guanylyl cyclases (pGCs) that modify GTP to elevate intracellular cGMP levels. Increases in cardiac cGMP levels inhibit ischemia/reperfusion damage by activation of the $\mathrm{Akt} / \mathrm{Bad} / \mathrm{Bcl} 2$ signaling pathway that prevents

Nonstandard abbreviations used: ANP, atrial natriuretic peptide; CHF, congestive heart failure; EGFP, enhanced GFP; GC, guanylyl cyclase; NES, nuclear exclusion sequence; NLS, nuclear localization sequence; pGC, particulate GC.

Conflict of interest: The authors have declared that no conflict of interest exists.

Citation for this article: J. Clin. Invest. 115:2716-2730 (2005).

doi:10.1172/JCI24280. apoptotic cell death (19). Protective effects of cGMP may involve downstream activation of cGMP-dependent PKG, expressed in cardiomyocytes as the PKG I (but not PKG II) isoform (20), since PKG activates PI3K/Akt kinase signaling in vascular smooth muscle and endothelial cells (21-25). The ANP/cGMP/PKG signaling cascade is well known for antiapoptotic activity in neuronal cell types (26) but is relatively unstudied in cardiomyocytes. However, ANPinduced cGMP signaling could certainly promote antiapoptotic responses in cardiomyocytes via Akt activation, since the protective effects of Akt in the myocardium are well known (27-29).

The mechanism(s) of Akt-mediated activation and antiapoptotic signaling in cardiomyocytes remain(s) enigmatic despite continued progress from multiple laboratories. Akt accumulates in the nucleus following stimulation with cardioprotective stimuli such as IGF-1 (30) or estrogen (31), suggesting that biologically relevant targets are located in the nuclear compartment. Our previous studies indicate that nuclear targeting of Akt exerts potent antiapoptotic action in the myocardium and isolated cardiomyocytes (32). Recently, a cytoskeletal focal adhesion component named zyxin was demonstrated to accumulate in the nucleus of vascular smooth muscle cells following mechanical stretch and redistribute in response to ANP or an active cGMP analog (33, 34). Intriguing properties of zyxin, such as an inherent nuclear exclusion sequence (NES), possession of multiple LIM-domain motifs sharing homology with transcription factors, and constitutive shuttling through the nucleus in cultured fibroblasts (35) all implicate this protein in nuclear-signal transduction. Collectively, these observations suggest that redistribution of zyxin from the cytoskeleton to the nucleus serves as a signaling mechanism for transmission of ANP-mediated effects. 
A

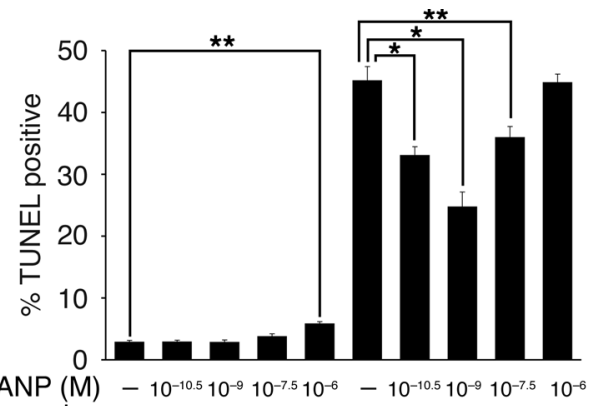

Staurosporine - -+-++++
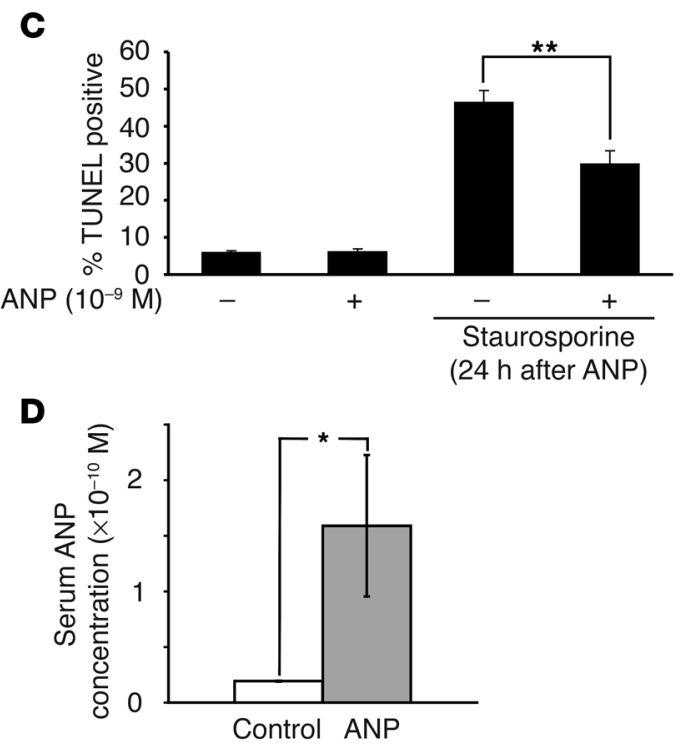

B

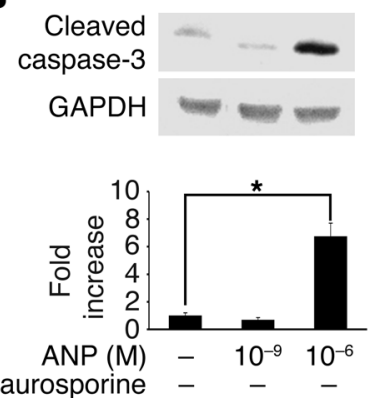

E

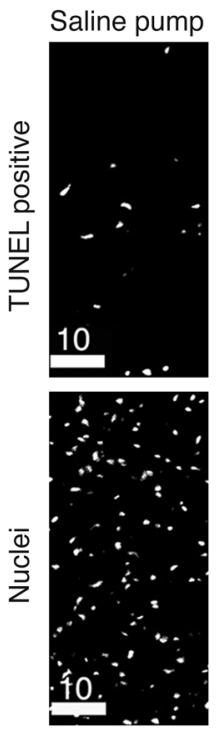

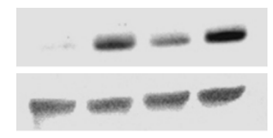
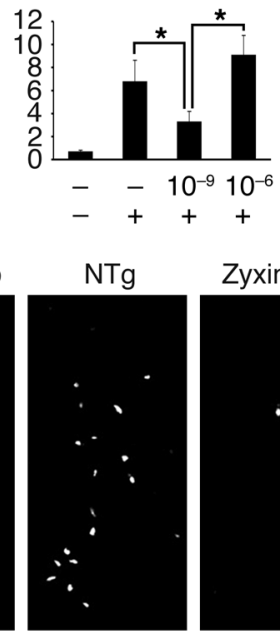

Zyxin-n.t.
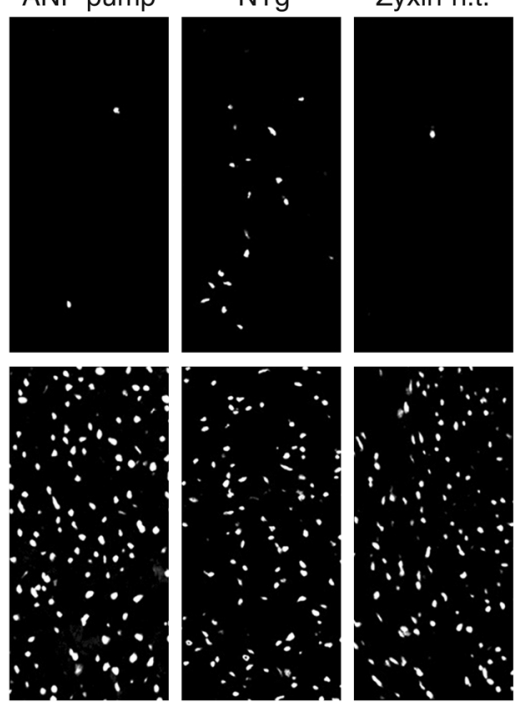
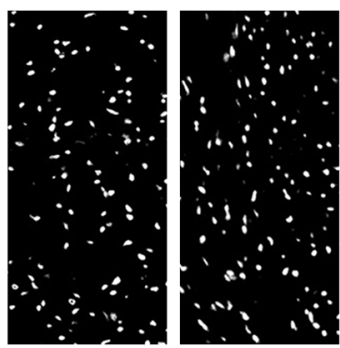

$\mathbf{F}$

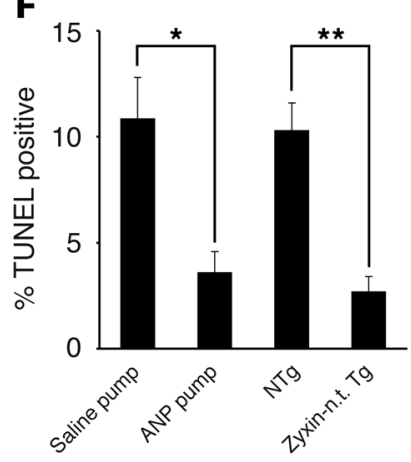

G

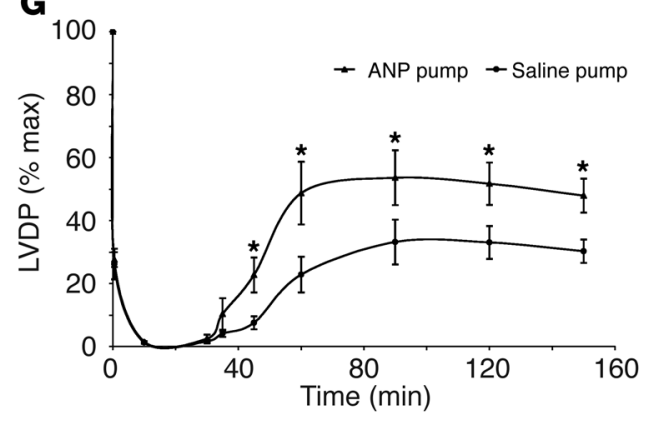

H

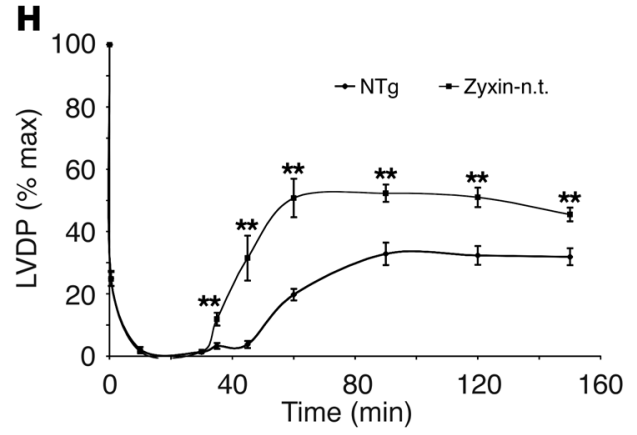

Figure 1

ANP exposure exerts concentration-dependent antiapoptotic effects upon cardiomyocytes and is protective in vivo. (A) Cardiomyocytes were plated onto chamber slides and preincubated with or without ANP (concentration range from $10^{-10.5} \mathrm{M}$ to $10^{-6} \mathrm{M}$ as indicated for 1 hour) followed by 2 hours of vehicle only $(-)$ or staurosporine treatment $(+; 1 \mu \mathrm{M})$. Apoptosis was assessed by TUNEL assay. (B) Apoptotic signaling in cultured cardiomyocytes evaluated by cleaved caspase-3. Quantitation of caspase-3 immunoblot was performed by densitometric analysis using GAPDH as a loading standard to correct for slight differences in protein loading. ANP treatment protocol was the same as in A. (C) Cardiomyocytes were treated with ANP for 24 hours prior to 2 hours apoptotic stimulus with staurosporine. Apoptosis was assessed by TUNEL assay. (D) Circulating serum levels of ANP from mice implanted with osmotic pumps as determined by ELISA assay (see Methods; $n=4$ for each group). (E-H) Analyses of hearts from mice implanted with osmotic pumps (saline control versus ANP treated) or genetically engineered to express nuclear-targeted zyxin (nontransgenic [NTg] control versus nuclear-targeted zyxin [zyxin-n.t.]) as described in Methods. (E) Representative confocal micrographs of TUNEL assays performed on myocardial sections of hearts subjected to ischemia/reperfusion damage. Scale bars: $10 \mu \mathrm{m}$. (F) Quantitation of TUNEL-positive nuclei from myocardial sections of mice as indicated. $(\mathbf{G}-\mathbf{H})$ Recovery of hemodynamic function during reperfusion phase for mouse groups receiving osmotic pump implants (G) or genetically engineered to express nuclear targeted zyxin $(\mathbf{H})$. ${ }^{*} P<0.05$ or ${ }^{* \star} P<0.01$ for each indicated comparison in $\mathbf{A}-\mathbf{H}$. $n=3$ for experiments in A-C; $n=4$ for experiments in D-H. LVDP, left ventricular developed pressure. 

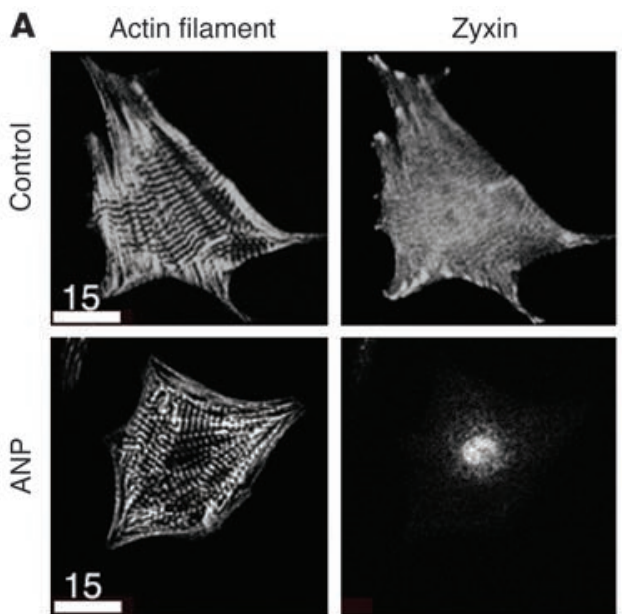

B

Control
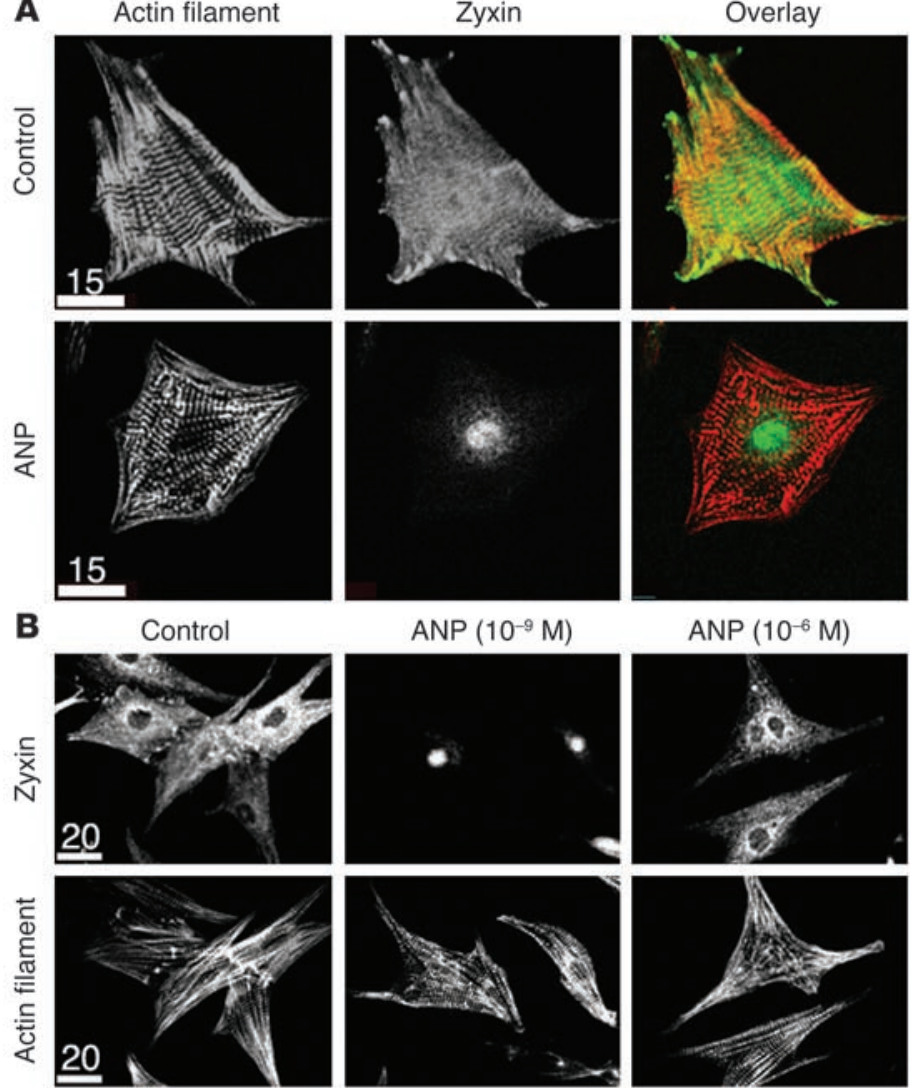

$\operatorname{ANP}\left(10^{-6} \mathrm{M}\right)$
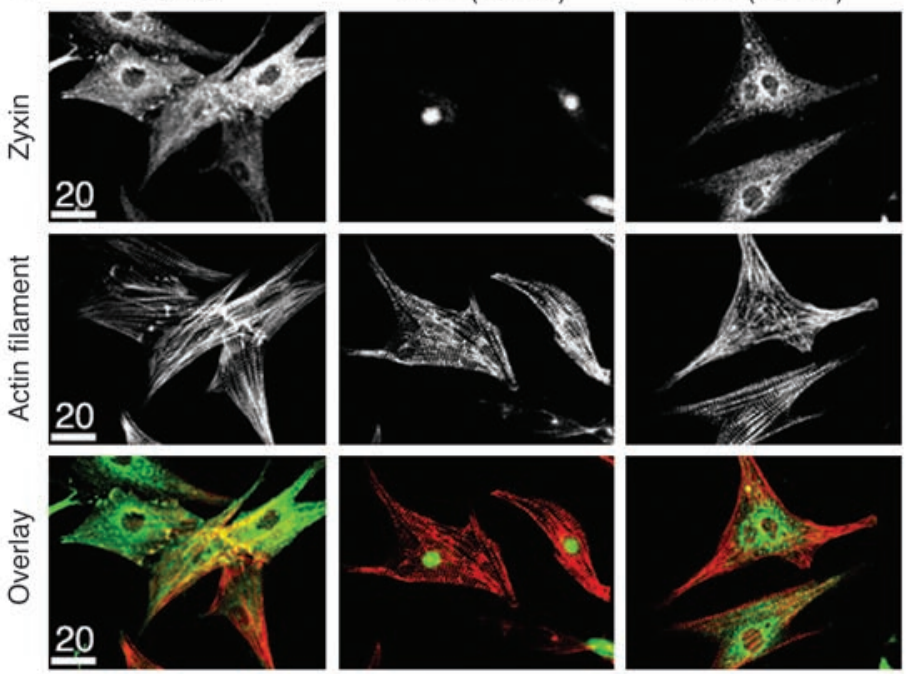
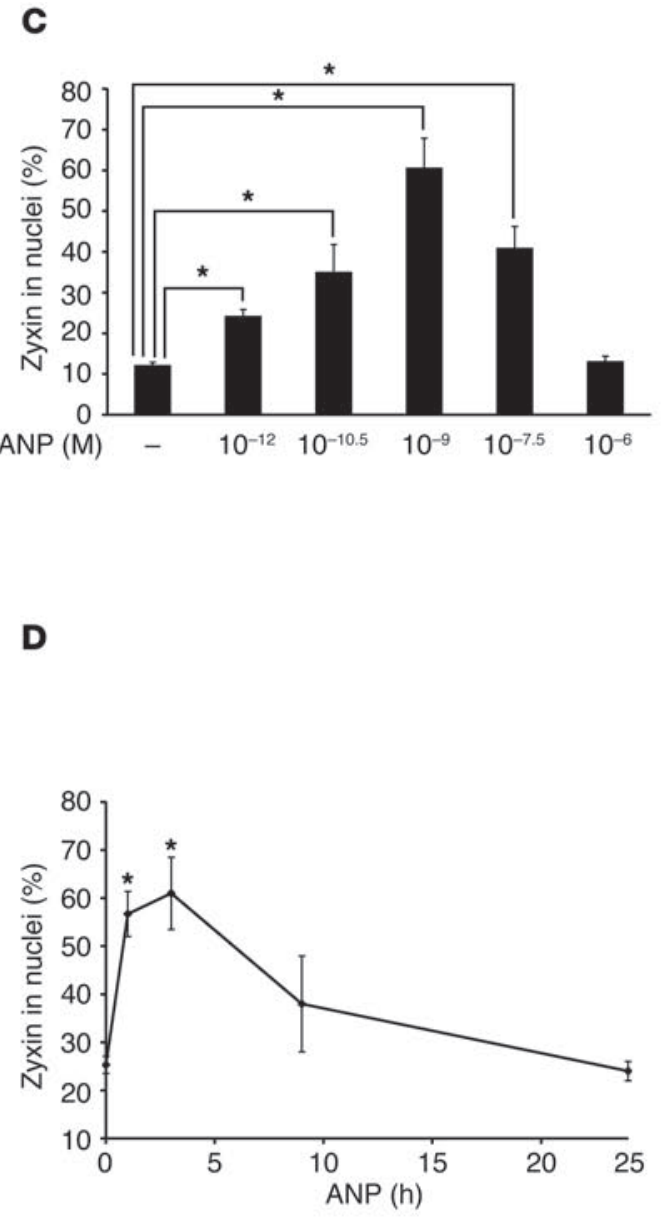

Figure 2

ANP induces nuclear accumulation of zyxin at antiapoptotic concentration. Immunolabeling (A and B) and quantitation of nuclear labeling (C and $\mathbf{D}$ ) in cultured cardiomyocytes treated with ANP $\left(10^{-9}\right.$ for 1 hour for $\mathbf{A}$, B, and $\mathbf{D} ; 10^{-6} \mathrm{M}$ for 1 hour also shown for $\left.\mathbf{B}\right)$. Immunolabeling at high (A) and low (B) magnification shows zyxin (green in overlay) predominantly associated with myofibrillar striations and focal adhesion regions in periphery of untreated cells (control). $10^{-9} \mathrm{M}$ ANP treatment resulted in redistribution and nuclear accumulation of zyxin whereas nuclear accumulation did not occur at $10^{-6} \mathrm{M}$ ANP. Myofibrillar organization was observed with phalloidin to label actin filaments (red in overlay). Bar measurements in micrographs represent distance in $\mu \mathrm{m}$. Quantitation (C) shows dosage-dependent nuclear accumulation of zyxin peak at $10^{-9} \mathrm{M}$ ANP 2 hours after treatment. Time course of nuclear zyxin accumulation (D) shows peak level within hours after treatment and return to normal level within 24 hours. $n=3$ for all experiments. ${ }^{\star} P<0.01$.

Results presented here reconcile ANP-related observations regarding cell survival with activation of cGMP-dependent signaling cascades leading to nuclear accumulation of Akt in cardiomyocytes. Furthermore, connections are established between ANP-mediated Akt activity and redistribution of zyxin from the cytoskeleton to the nucleus. Delineating the signaling mechanisms for ANP-mediated antiapoptotic activity mediated through cGMP-dependent Akt activation in cardiomyocytes is an important factor in understanding therapeutic applications of natriuretic peptides in the treatment of heart failure.

\section{Results}

Antiapoptotic effect of ANP exposure upon cardiomyocytes varies with concentration. Cultured cardiomyocytes were pretreated with vehicle or ANP at concentrations of $10^{-10.5}, 10^{-9}, 10^{-7.5}$, or $10^{-6} \mathrm{M}$ followed by 2-hour exposure to apoptotic stimulation with $1 \mu \mathrm{M}$ staurosporine treatment. DNA cleavage in cells undergoing apoptosis was detected by TUNEL assay in conjunction with confocal microscopy. ANP treatment produced significantly different effects based upon concentration. ANP concentrations between $10^{-10.5}$ and $10^{-7.5} \mathrm{M}$ were antiapoptotic, protecting cardiomyocytes from staurosporine challenge and decreasing TUNEL-positive nuclei compared with controls (Figure 1A), although the protective effect was maximal at a low therapeutic dose of $10^{-9} \mathrm{M}$. In contrast, a higher dose of ANP $\left(10^{-6} \mathrm{M}\right)$ increased TUNEL-positive nuclei by 2 -fold $(202 \% \pm 11.2 \%)$ in cardiomyocytes without any additional apoptotic stimuli. The proapoptotic effect of ANP at high concentrations was confirmed by demonstration of a 7 - fold increase in cleaved caspase- 3 relative to that in vehicle-treated control cell cultures (Figure 1B). 
A

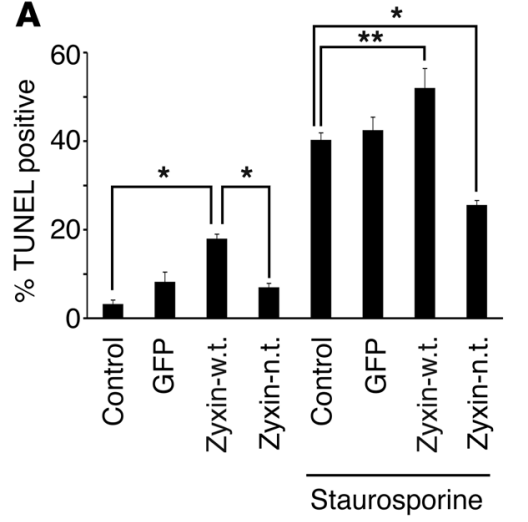

B

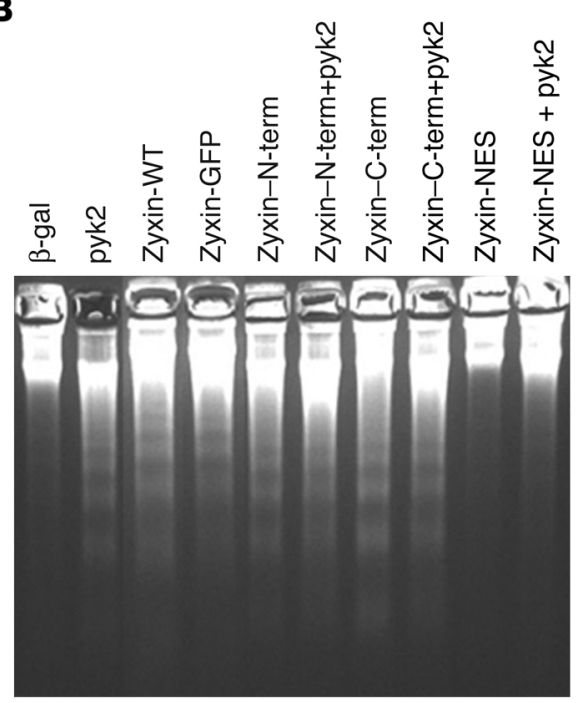

C

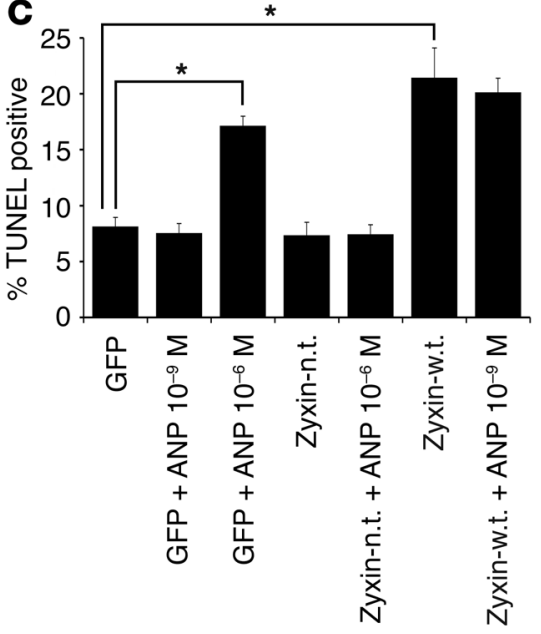

\section{Figure 3}

Nuclear accumulation of zyxin is antiapoptotic. TUNEL assay (A) of cultured cardiomyocytes infected with adenoviruses expressing GFP, fulllength zyxin (zyxin-w.t.), or nuclear-targeted zyxin for 2 hours followed by treatment with 1 umol/l staurosporine to induce apoptosis. Results shown were derived from 3 separate experiments. (B) Apoptosis in cultured cardiomyocyte evaluated by DNA laddering. Adenoviruses expressing $\beta$-gal or zyxin as wild-type, GFP-conjugated full length (GFP), residues 1-322 (N-term), residues 349-542 (C-term), or NES-deleted residues 322-331 (NES). Apoptotic stimulation was initiated by overexpression of pyk2 kinase as previously described (35). Typical result is shown of 3 separate repetitions for the laddering experiment. (C) TUNEL assay of cultured cardiomyocytes shows protective effect of nuclear-targeted zyxin accumulation in response to apoptotic challenge by ANP $\left(10^{-6} \mathrm{M}\right)$ but lack of protection by ANP $\left(10^{-9} \mathrm{M}\right)$ in response to apoptotic challenge by full-length wild-type zyxin accumulation. Adenoviral vectors were used for expression of zyxin constructs with GFP expression shown as a control for effects of ANP treatments. ${ }^{*} P<0.01$; ${ }^{*} P<0.05$.

Although $10^{-6} \mathrm{M}$ ANP alone increased accumulation of cleaved caspase- 3 without staurosporine, the same concentration found effective at lowering TUNEL labeling $\left(10^{-9} \mathrm{M}\right)$ also protected against staurosporine challenge and decreased caspase- 3 cleavage (Figure 1B). Protective effects of $10^{-9} \mathrm{M}$ ANP against staurosporine-induced cell death persisted for up to 24 hours following treatment (Figure 1C), indicating that triggering of survival signaling by ANP promotes prolonged antiapoptotic effects. Collectively, these results indicate that ANP inhibits or promotes cardiomyocyte apoptosis depending upon concentration. ANP-mediated protective effects were confirmed in the intact myocardium using an ex vivo isolated heart model of ischemia/reperfusion damage. Mice implanted with an osmotic pump exhibited ANP serum concentrations of $1.6 \times 10^{-10}$ at time of sacrifice (Figure 1D), when hearts were harvested and challenged with ischemia/reperfusion injury. ANP treatment reduced apoptotic cell death quantitated with TUNEL assays by $68.8 \%$ compared with that in control hearts from mice receiving osmotic pumps with vehicle alone (Figure 1, $\mathrm{E}$ and $\mathrm{F}$ ). Furthermore, hemodynamic assessments on hearts from mice treated with ANP showed improved functional recovery following reperfusion (Figure 1G; 2.1-fold higher left ventriculardeveloped pressure at 60 minutes).

ANP induces nuclear accumulation of zyxin at antiapoptotic concentrations. Cultured cardiomyocytes were exposed to ANP at concentrations found to either promote or inhibit apoptosis $\left(10^{-6} \mathrm{M}\right.$ versus $10^{-9} \mathrm{M}$, respectively; Figure 1) to determine the effect upon intracellular distribution of zyxin. Zyxin is localized along myofibrils and concentrated at focal adhesions in unstimulated cardiomyocytes (Figure 2, A and B, top panels), consistent with the role of this protein as a cytoskeletal-membrane constituent. Zyxin undergoes a marked intracellular redistribution in response to treatment of cells with $10^{-9} \mathrm{M}$ ANP, characterized by translocation and accumulation in the nucleus (Figure 2, A and B). Nuclear accumulation of zyxin occurs in a majority of the cells at ANP concentrations of $10^{-9} \mathrm{M}$ but not at the higher dose of $10^{-6} \mathrm{M}$ (Figure 2B) in a concentration-dependent fashion (Figure 2C). The induction of zyxin nuclear accumulation by ANP peaks within 5 hours after exposure and returns to basal levels within 24 hours after treatment (Figure 2D). These results indicate that ANP can promote nuclear accumulation of zyxin at the same concentration found to exert antiapoptotic signaling in cultured cardiomyocytes. Furthermore, a higher concentration of ANP found to be proapoptotic $\left(10^{-6} \mathrm{M}\right)$ does not promote nuclear accumulation of zyxin.

Nuclear accumulation of zyxin is antiapoptotic. The association between antiapoptotic action of ANP and nuclear accumulation of zyxin suggests a potential mechanism for protective cell signaling via nuclear zyxin accumulation. This postulate was tested by constructing adenoviruses expressing zyxin either as full-length wild-type or full-length nuclear-targeted protein versions. The efficacy of these zyxin constructs in inhibiting cardiomyocyte apoptosis in response to staurosporine challenge was examined by both TUNEL assay and DNA laddering (Figure 3, A and B, respectively). Nuclear targeting of zyxin in TUNEL assays was accomplished by fusion of the zyxin cDNA to a multimerized series of nuclear localization sequences at the $\mathrm{C}$ terminus of the molecule. Comparison of the TUNEL results showed that full-length zyxin promoted DNA cleavage but that nuclear targeting of zyxin abrogated the increase in TUNEL labeling (Figure 3A). Furthermore, TUNEL assay results showed that the nuclear-targeted zyxin construct significantly inhibited staurosporine-induced DNA cleavage. This protective effect was unique to the 

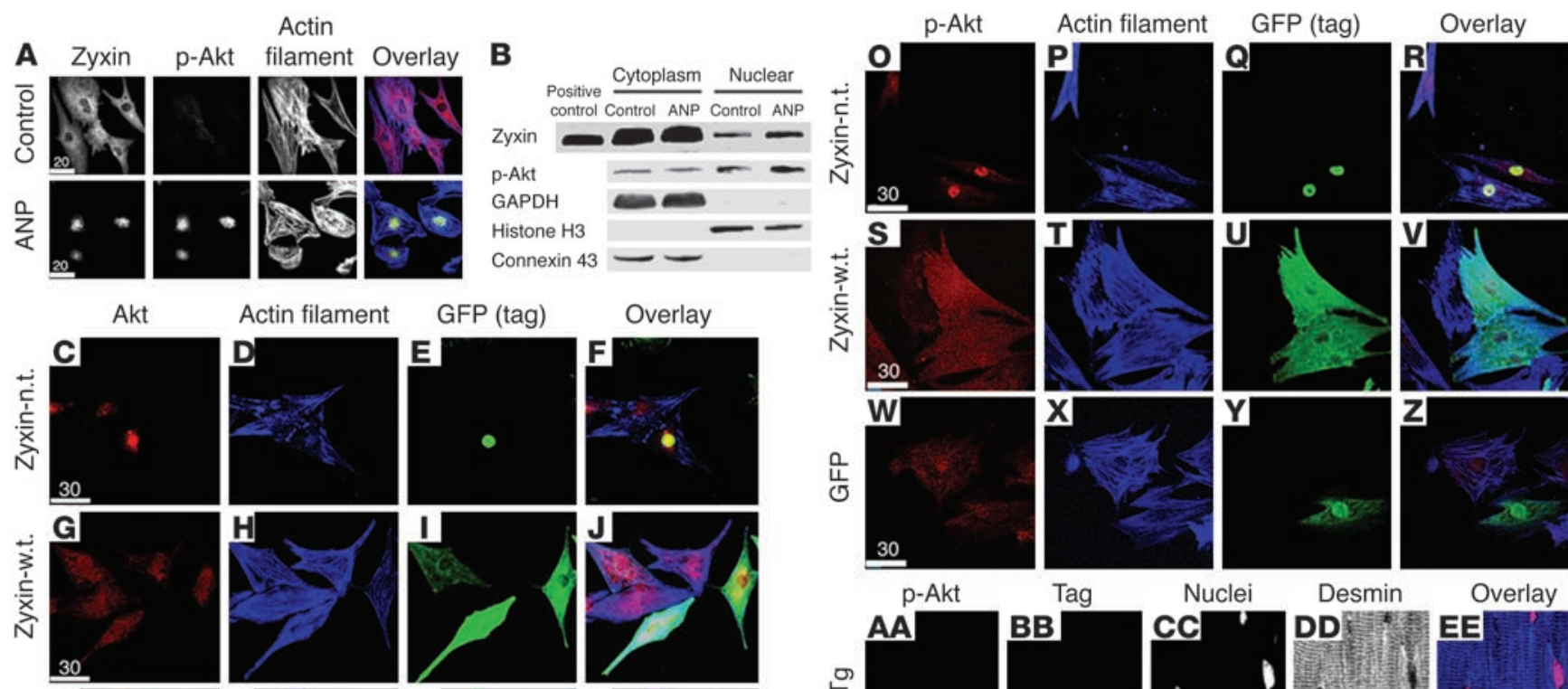

Overlay
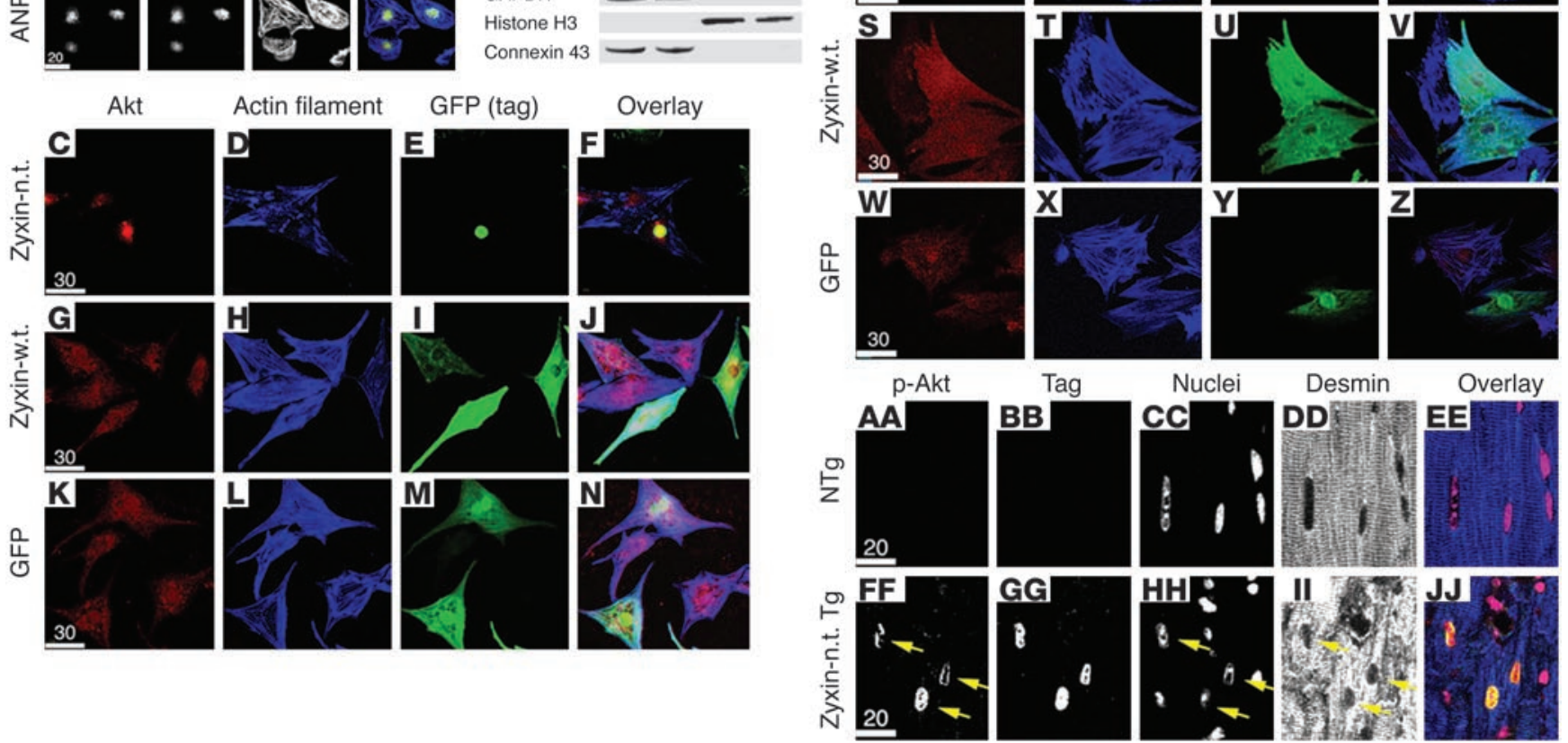

Figure 4

Nuclear accumulation of activated Akt kinase in response to ANP treatment or expression of nuclear-targeted zyxin. Cultured cardiomyocytes $(\mathbf{A}-\mathbf{Z})$ or myocardial sections (AA-JJ) showing nuclear accumulation of Akt. Confocal micrographs (A) and immunoblot analysis (B) of myocytes treated with vehicle (control) or ANP $\left(10^{-6} \mathrm{M}\right)$ show nuclear accumulation of both zyxin and phospho-Akt ${ }^{473}$ (p-Akt) in response to ANP exposure. Overlay channels in A correspond to zyxin (red), phospho-Akt (green), and actin filaments to show myofibril organization (blue). In B, immunoblots for GAPDH, histone $\mathrm{H3}$, and connexin 43 demonstrate subcellular fractionation of the cytosolic, nuclear, and membrane fractions, respectively. Also shown are confocal micrographs of cardiomyocytes labeled with antibodies (shown in red) to either total Akt (C-N) or phospho-Akt ${ }^{473}(\mathbf{O}-\mathbf{Z})$. Cells were infected with adenovirus (shown in green) expressing GFP (K-N and $\left.\mathbf{W}-\mathbf{Z}\right)$, nuclear-targeted zyxin (C-F and $\mathbf{O}-\mathbf{R}$ ), or full-length zyxin (G-J and $\mathbf{S}-\mathbf{V})$. Myofibrillar organization was observed with phalloidin to label actin filaments (shown in blue). (C-N) Akt immunoreactivity was mainly observed in cytoplasm in cultures expressing full-length zyxin or GFP but accumulated in the nucleus following expression of nuclear-targeted zyxin virus. (O-Z) Phospho-Akt ${ }^{473}$ immunoreactivity was observed at low levels in cells expressing either fulllength zyxin or GFP but accumulated in the nucleus following expression of nuclear-targeted zyxin. Myocardial sections (AA-JJ) show nuclear accumulation of phospho-Akt ${ }^{473}$ in transgenic mice expressing cardiac-specific nuclear-targeted zyxin (zyxin-n.t. Tg) but not in nontransgenic control samples. Arrows indicate nuclei positive for both zyxin and phospho-Akt ${ }^{473}$. Transgenic zyxin was visualized using antibody to myc-tag (tag) and desmin to show myofibrillar structure. Overlay colors correspond to phospho-Akt 473 (red), myc-tag (green), nuclei (pink), and desmin (blue) with coincidence of red and blue labeling appearing yellow. Scale bars: $20 \mu \mathrm{m}$ (A and AA-JJ); $30 \mu \mathrm{m}$ (C-Z).

nuclear-targeted zyxin construct as uninfected, GFP-expressing, and full-length zyxin-expressing cardiomyocytes all showed comparable TUNEL labeling following staurosporine challenge. The combination of staurosporine and full-length zyxin together showed the highest level of TUNEL labeling of all conditions examined, clearly demonstrating that cytoplasmic accumulation of zyxin was not protective in response to staurosporine challenge (Figure 3A). DNA laddering assays (Figure 3B) confirmed and extended the results obtained by TUNEL assays by using additional adenoviral constructs of zyxin. Adenoviruses tested in the DNA laddering assay included full-length wild-type zyxin, GFP-tagged zyxin, the region of zyxin prior to the endogenous NES (amino-terminal residues 1-321), the LIM-domain region of zyxin following endogenous NES (carboxylterminal residues 349-542), and a construct lacking endogenous NES-deleted residues 322-331) previously reported to accumulate in the nucleus (36). Apoptotic stimulation in DNA laddering experi- ments was provided by overexpression of pyk2 kinase that had been previously shown to be apoptotic in cultured cardiomyocytes (37). Consistent with previously observed TUNEL assay results, the cytoplasmic accumulation of zyxin, either in full-length or truncated form, promoted DNA cleavage either alone or in combination with pyk2 (Figure 3B). In contrast, the NES-deleted zyxin construct that accumulated in the nucleus was the only overexpressed protein to inhibit pyk2-induced apoptosis. Assessing the balance between ANP exposure and zyxin accumulation, nuclear-targeted zyxin expression inhibited the proapoptotic effect of $10^{-6} \mathrm{M}$ ANP exposure, but stimulation of cells with $10^{-9} \mathrm{M}$ ANP failed to inhibit apoptotic cell death induced by overexpression of wild-type zyxin (Figure 3C). Apoptotic signaling induced by the accumulation of overexpressed wild-type zyxin was apparently too strong to be overcome by ANP administration operating at near-physiologic concentration. The antiapoptotic effect of nuclear-targeted zyxin was confirmed in vivo using hearts 
A
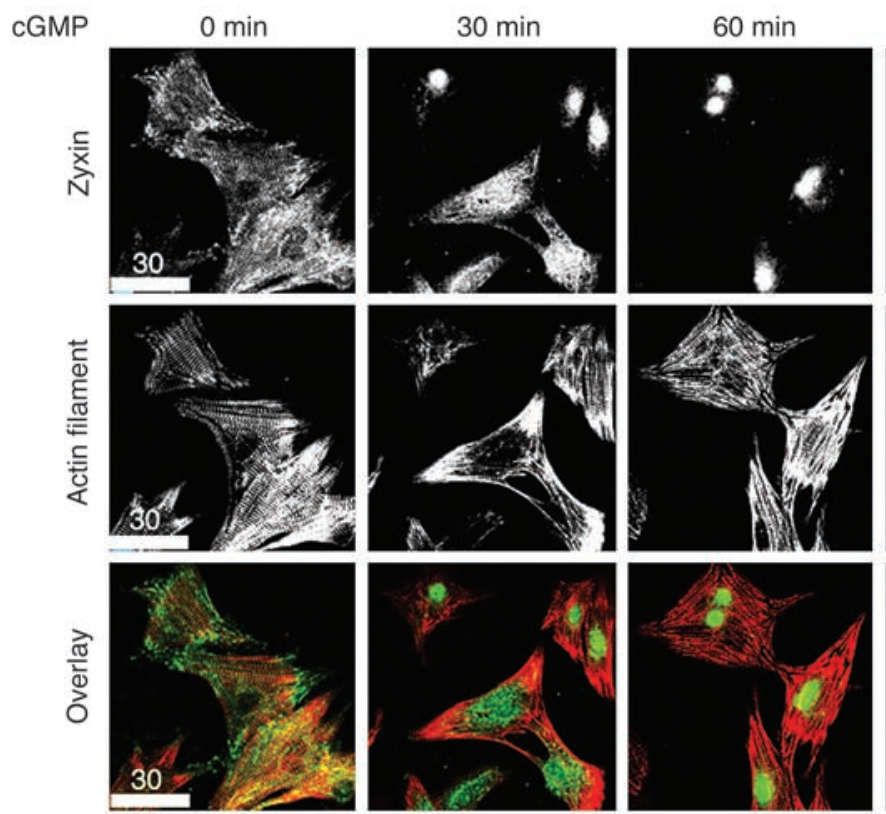

B
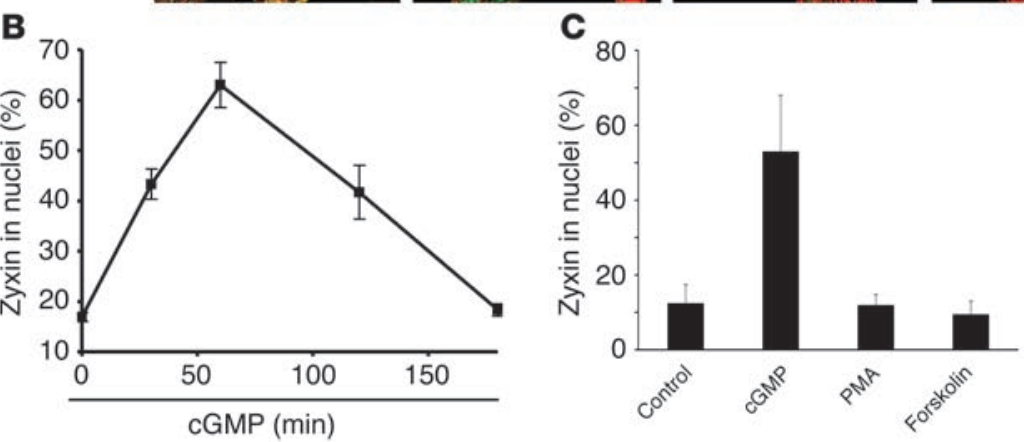

$120 \mathrm{~min}$
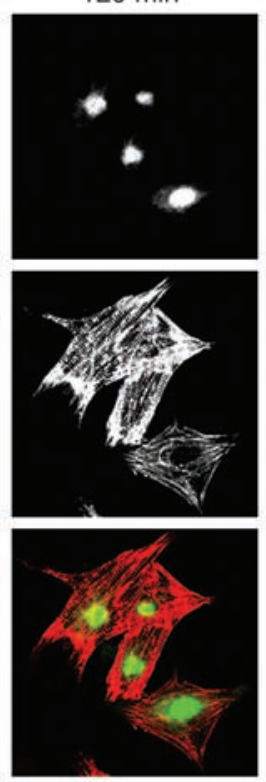

D
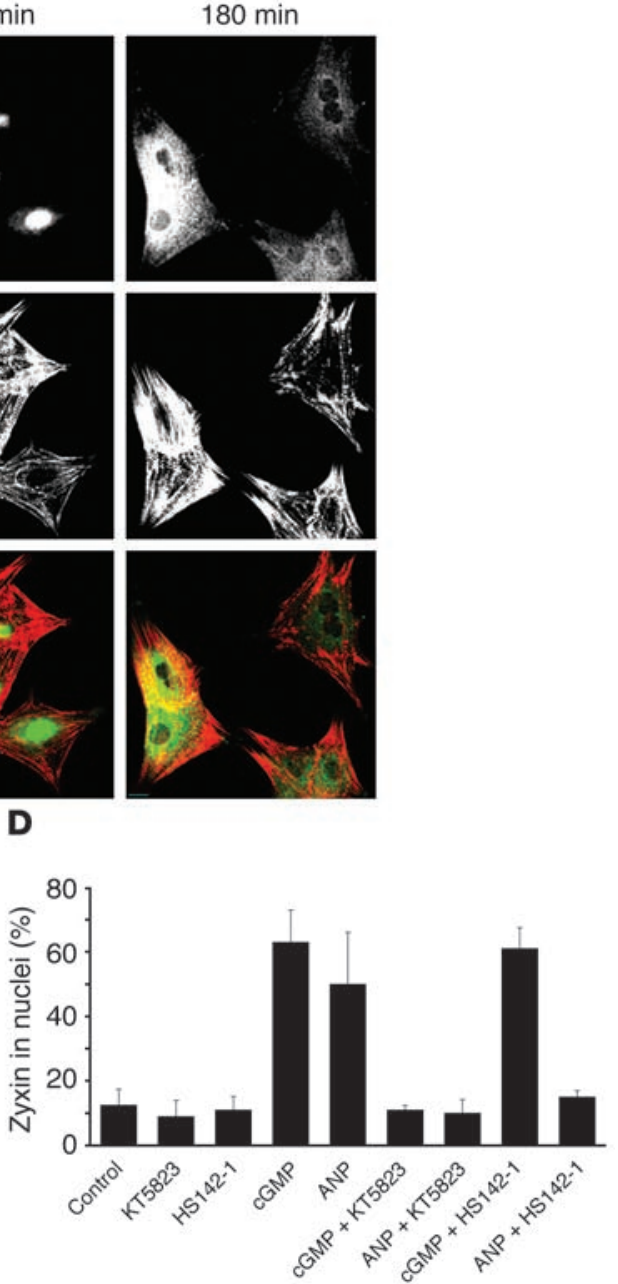

Figure 5

Nuclear accumulation of zyxin is mediated by cGMP-dependent signaling. Time course of zyxin nuclear accumulation in cultured cardiomyocytes treated with cGMP $\left(10^{-4} \mathrm{M}\right)$. (A) Confocal microscopy shows distribution of zyxin (green in overlay) and myofibrils (red in overlay) following exposure to cGMP. Cells were labeled with phalloidin (actin filament) to reveal sarcomeric organization. Scale bars: $30 \mu \mathrm{m}$. (B) Time course of nuclear zyxin accumulation shows peak level within minutes after treatment and return to normal level within 3 hours. (C) Activation of PKA or PKC signaling did not induce zyxin nuclear translocation. Bar graph shows the ratio of zyxin nuclear translocation with cGMP $\left(10^{-4} \mathrm{M}\right), \mathrm{PMA}(10 \mathrm{ng} / \mathrm{ml})$, or forskolin $\left(10^{-5} \mathrm{M}\right)$ treatment for 1 hour as assessed by confocal microscopy. (D) Participation of ANP or PKG in nuclear translocation of zyxin was demonstrated by use of HS142-1 (ANP receptor inhibitor; $10 \mu \mathrm{g} / \mathrm{ml}$ ) or KT5823 (PKG inhibitor; $5 \times 10^{-6} \mathrm{M}$ ). Bar graph shows the ratio of zyxin nuclear translocation with or without inhibitor pretreatment followed by stimulation with cGMP $\left(10^{-4} \mathrm{M}\right)$ or ANP $\left(10^{-9} \mathrm{M}\right) . n=3$ for all experiments.

from transgenic mice. Myocardial-specific transgenic expression of nuclear-targeted zyxin significantly inhibited TUNEL labeling of nuclei in sections of hearts challenged by ischemia/reperfusion damage (Figure $1, \mathrm{E}$ and $\mathrm{F}$ ) and improved recovery of contractile function during the reperfusion phase (Figure $1 \mathrm{H})$. Collectively, these findings are consistent with nuclear accumulation of zyxin mediating antiapoptotic effects in cardiomyocytes, consistent with the postulate that zyxin is involved in protective effects mediated by ANP.

Nuclear accumulation of zyxin induces nuclear accumulation of activated Akt kinase. Antiapoptotic effects mediated by nuclear accumulation of zyxin (Figure 2) are reminiscent of the survival kinase Akt that accumulates in the nucleus and inhibits apoptosis (33). This circumstantial correlation was pursued in experiments to determine if nuclear accumulation of zyxin prompted coincident nuclear accumulation of activated Akt. Cultured cardiomyocytes stimulated with
$10^{-9} \mathrm{M}$ ANP showed coincident nuclear accumulation of both zyxin and phospho-Akt ${ }^{473}$ by immunofluorescence microscopy (Figure 4A) and increased partitioning of both zyxin and phospho-Akt ${ }^{473}$ to the nuclear fraction by immunoblot analyses (Figure 4B). Appropriate partitioning of cellular components was checked by immunolabeling with antibodies to connexin 43 (cell membrane) and histone (nucleus). Confocal microscopy of cardiomyocytes infected with adenoviruses expressing GFP alone, GFP-tagged full-length zyxin, or GFP-tagged nuclear-targeted zyxin showed that nuclear accumulation of GFP-tagged nuclear-targeted zyxin promoted concurrent nuclear accumulation of Akt (Figure 4, C-N). Furthermore, similar analyses using an antibody against phospho- $\mathrm{Akt}^{473}$ showed that nuclear-localized Akt was phosphorylated, indicative of kinase activation (Figure 4, O-Z). In contrast, overexpression of either GFP alone or GFP-tagged full-length zyxin did not promote nuclear 


\section{A Actin filament}
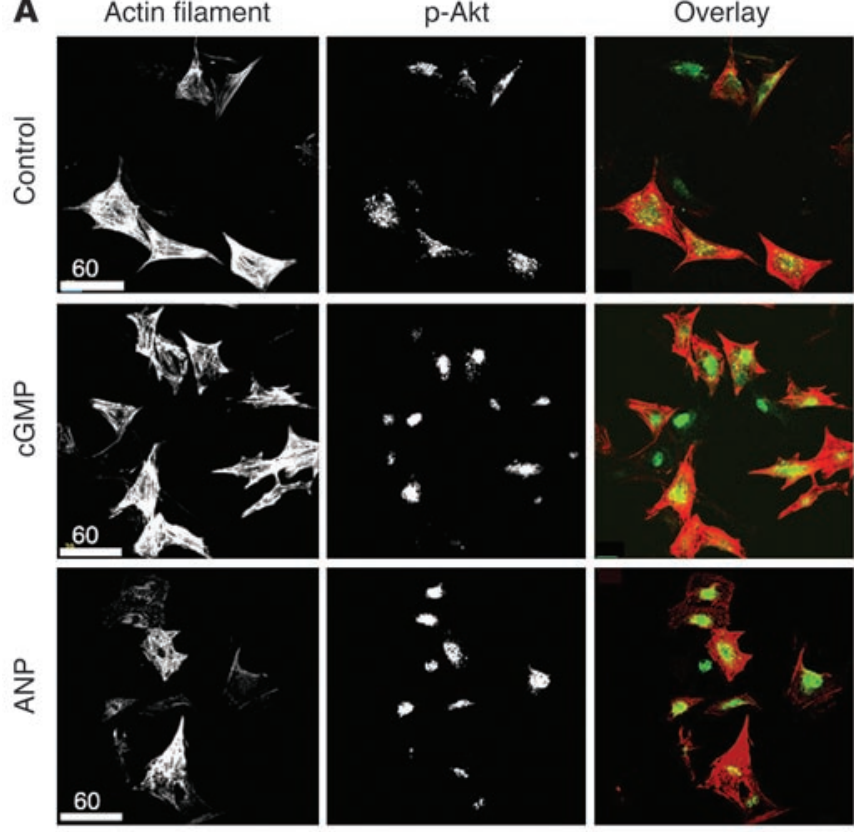

B
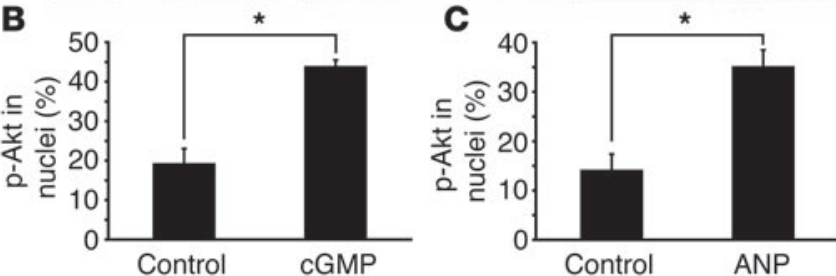

Figure 6

Elevation of cGMP induces rapid nuclear accumulation of activated Akt kinase. cGMP and ANP increased the expression level of phospho-Akt in the nuclear fraction. Cells were labeled with phalloidin ( $\mathbf{A}$, left side) to reveal sarcomeric organization. Confocal microscopy shows the nuclear accumulation of phospho-Akt increase in the cardiomyocyte with cGMP $\left(10^{-4} \mathrm{M}\right)$ or ANP $\left(10^{-9} \mathrm{M}\right)$ treatment $(\mathbf{A}$, middle). Actin filament (red) and zyxin (green) are depicted in overlay panels (A, right side). Scale bars, $60 \mu \mathrm{m}$. (B and C) Bar graph shows the ratio of the nuclear accumulation of phospho-Akt with cGMP $\left(10^{-4} \mathrm{M}\right)$ or ANP $\left(10^{-9} \mathrm{M}\right)$ treatment obtained from confocal microscopic analysis. $n=3 .{ }^{*} P<0.05$.

accumulation of Akt. Promotion of nuclear Akt accumulation by nuclear-targeted zyxin was confirmed in vivo using hearts from transgenic mice. Myocardial-specific transgenic expression of nuclear-targeted zyxin increased nuclear labeling for phospho-Akt ${ }^{473}$ as demonstrated by confocal microscopy (Figure 4, AA-JJ). Together, these results indicate that nuclear accumulation of activated Akt kinase is likely to play an important role in the antiapoptotic effects resulting from nuclear accumulation of zyxin.

Nuclear accumulation of zyxin is mediated by cGMP-dependent signaling. Circumstantial associations exist between ANP-mediated effects and activation of cGMP-dependent signaling (26). To establish a cGMP-dependent mechanism for ANP-mediated effects upon zyxin, cultured cardiomyocytes were treated with $10^{-4} \mathrm{M}$ 8-bromo-cGMP (a cell-permeable cGMP analogue) and analyzed by confocal microscopy. Zyxin nuclear translocation occurred within minutes after initiation of cGMP signaling, peaked after 1 hour, and returned to levels comparable to basal stimulation within 2 hours (Figure 5, A and B). Nuclear accumulation of zyxin was unaffected by activators of
cAMP-dependent (forskolin) or PKC-dependent (PMA) signaling at the 1-hour peak time point (Figure 5C and Supplemental Figure $\mathrm{S} 1$; supplemental material available online with this article; doi:10.1172/JCI24280DS1), suggesting the effect is specific to cGMP. Involvement of ANP receptor-mediated signaling in promotion of zyxin nuclear accumulation was confirmed using HS142-1 (an ANP receptor inhibitor), which blocked nuclear accumulation of zyxin when cultures were treated with the inhibitor prior to ANP exposure (Figure 5D) but alone had no effect upon zyxin localization (Supplemental Figure S2). However, HS-142-1 did not block zyxin accumulation in the nucleus initiated by cell-permeant cGMP, indicating that cGMP lies downstream of ANP-receptor activation. cGMP-dependent signaling in mediation of zyxin nuclear accumulation was confirmed using KT5823 (a specific PKG inhibitor), which alone had no effect upon zyxin localization (Supplemental Figure S2) but abrogated nuclear accumulation of zyxin when cultures were treated with the inhibitor prior to cGMP or ANP exposure (Figure 5D). These results implicate a cGMP-dependent pathway through natriuretic peptide receptor signaling in the nuclear accumulation of zyxin mediated by ANP treatment.

Elevation of CGMP or ANP treatment induces rapid nuclear accumulation of activated Akt kinase. The connection between ANP, zyxin, and Akt (Figures 2-4) in conjunction with dependence of zyxin nuclear accumulation upon cGMP-dependent signaling (Figure 5) suggests that nuclear accumulation of activated Akt also involves cGMP-mediated signaling. The association of cGMP-mediated signaling and nuclear accumulation of Akt was demonstrated by confocal microscopy of cultured cardiomyocytes treated with cell-permeant cGMP analog for 1 hour (Figure 6A). Quantitation showed more than a 2-fold increase in phospho-Akt $\mathrm{At3}^{473}$ nuclear accumulation following cGMP treatment that was statistically significant (Figure 6B; $2.3 \pm 0.31, P<0.05)$. Similarly, treatment of the cultures with ANP for 1 hour also significantly increased nuclear-localized phospho-Akt ${ }^{473}$ more than 2-fold (Figure 6C; $2.5 \pm 0.24$ fold, $P<0.05)$. Collectively, these results establish induction of nuclear Akt accumulation by cGMP-dependent signaling and support the connection between ANP-mediated protective effects and cGMP-dependent accumulation of Akt in the nucleus.

Antiapoptotic effect of cGMP exposure varies with concentration. ANP exerted opposing effects upon cell survival depending upon concentration (Figure 1) that could be related to the intensity of cGMP-dependent signaling activity initiated by ANP. Effects of varying cGMP concentrations upon apoptotic cell death were assessed by TUNEL assay using cultured cardiomyocytes. Treatment with cell-permeant cGMP produced significantly differing effects based upon concentration. cGMP at a low concentration of $10^{-4} \mathrm{M}$ was antiapoptotic, protecting cardiomyocytes from the staurosporine challenge and decreasing TUNEL-positive nuclei by $37.8 \% \pm 4.4 \%$ compared with that of controls (Figure 7). In contrast, a higher dose of cGMP alone at $10^{-2} \mathrm{M}$ increased TUNEL-positive nuclei over 3 -fold $(320 \% \pm 7.6 \%)$ and at this higher concentration was ineffective at lowering TUNEL-positive cells in response to staurosporine challenge. Collectively, these results indicate that, like ANP, cGMP acts to inhibit or promote cardiomyocyte apoptosis depending upon concentration.

Cardioprotective stimuli induce nuclear accumulation of zyxin. In addition to ANP, other paracrine factors touted as cardioprotective agents are known to increase cGMP levels (38-40). Since nuclear 


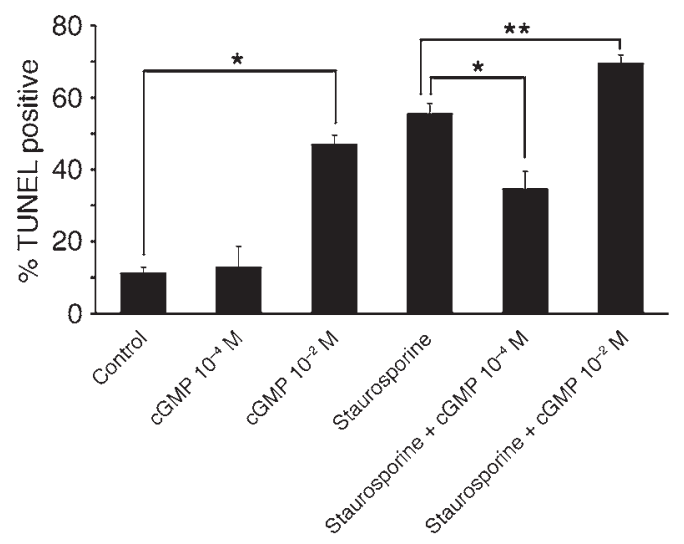

accumulation of zyxin is ANP- and cGMP-dependent (Figure 5), the abilities of various cardioprotective agents to mediate nuclear accumulation of zyxin were examined by confocal microscopy. The time course for nuclear zyxin accumulation resulting from treatment of cultured cardiomyocytes with IGF-1, adrenomedullin, and estradiol are shown in Figure 8. In comparison to the effect of ANP (Figure 2D), IGF showed a similar strong and rapid induction of nuclear zyxin accumulation in over $65 \%$ of cells that decayed over a 24-hour period. A similar cycle of rapid induction and decay occurred with adrenomedullin, albeit with a somewhat weaker response of slightly above $40 \%$ positive nuclei. Estradiol treatment produced a unique biphasic change in nuclear accumulation of zyxin that differed from the other 3 factors tested. Recurrence of nuclear zyxin accumulation in the later phase of estradiol treatment may stem from autocrine or paracrine action of ANP induced by estrogen stimulation (39). Treatment with the PKG inhibitor KT 5823 blocked nuclear accumulation of zyxin induced by any of these cardioprotective stimuli (Figure 8D). These results indicate that the nuclear accumulation of zyxin is a general characteristic of multiple cardioprotective stimuli that is dependent upon PKG activity, although differences in the kinetics and intensity of nuclear accumulation occur depending upon the inducing agent used.

ANP induces nuclear accumulation of zyxin in the myocardium. Observations of cGMP- and ANP-mediated nuclear accumulation in cultured cardiomyocytes were reproduced in the myocardium of normal mice as assessed by confocal microscopy and immunoblot analyses (Figure 9). ANP was administered continuously for 24 hours through implantation of a subcutaneous osmotic pump identical to the protocol followed for TUNEL assays (Figure 1, E and F) and myocardial sections examined by confocal microscopy. Zyxin is distributed in costameres of cardiomyocytes with a striated pattern predominantly coincident with sarcomeric desmin and is not detectable in nuclei of vehicle-treated control hearts but could be observed in nuclei of mice receiving ANP treatment (Figure 9, $\mathrm{A}-\mathrm{H}$, arrows) although desmin labeling remained unaffected. Furthermore, transgenic mice with cardiac-specific expression of constitutively active GC (41) also showed increased nuclear accumulation of zyxin (Figure 9, I-P), implicating cGMP-mediated signaling in the promotion of zyxin nuclear accumulation. Observation of nuclear phospho-Akt ${ }^{473}$ immunoreactivity showed induction of nuclear localization comparable to that observed for zyxin. Myocardial sections of nontransgenic control mice receiving the ANP osmotic pump treatment showed increased nuclear labeling for phospho-Akt ${ }^{473}$ (Figure 9, Q-BB, arrows). Comparable results

\section{Figure 7}

Antiapoptotic effect of cGMP exposure varies with concentration. TUNEL assay shows effect of cGMP treatment upon apoptosis induced by staurosporine $(1 \mu \mathrm{M})$ in cultured cardiomyocytes at low $\left(10^{-4}\right)$ or high $\left(10^{-2}\right)$ concentration. ${ }^{*} P<0.01 ;{ }^{* \star} P<0.05$.

yielding increased nuclear phospho-Akt ${ }^{473}$ labeling were obtained by bolus injection of cGMP (Figure 9, Q-BB, arrows). In addition, nuclear labeling for phospho-Akt ${ }^{473}$ was increased in transgenic mice with cardiac-specific expression of constitutively active GC (Figure 9, CC-JJ, arrows). Myocardial protein lysates processed for nuclear fractionation showed that ANP osmotic pump treatment increased nuclear accumulation of zyxin and phospho-Akt ${ }^{473}$ (Figure 9KK). Thus, zyxin and phospho-Akt ${ }^{473}$ nuclear accumulation in vivo responds to the same inductive stimuli that promote nuclear accumulation of these proteins in cultured cardiomyocytes in association with elevated cGMP signaling.

Cardioprotective stimuli induce association of activated Akt with zyxin. Coincidence of nuclear accumulation between zyxin and Akt (Figure 4) together with similar actions of ANP or cGMP to promote nuclear accumulation (Figures 2 and 5) suggest the potential for association between zyxin and activated Akt kinase. Formation of a multimolecular complex involving zyxin and Akt was assessed by immunoprecipitation using antibodies against phospho-Akt $\mathrm{t}^{47}$ followed by subsequent immunoblot detection for zyxin. No such complex between zyxin and Akt could be detected in control vehicle-treated cardiomyocytes
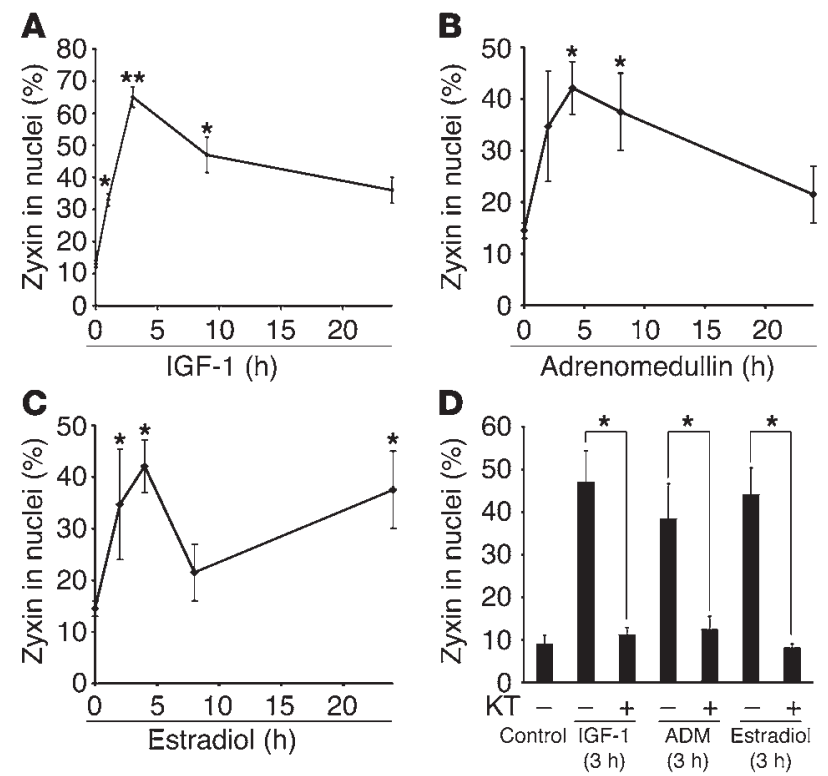

\section{Figure 8}

Cardioprotective stimuli induce nuclear accumulation of zyxin that depends upon PKG activity. Time course of zyxin nuclear accumulation following treatment of cultured cardiomyocytes with various antiapoptotic agents as indicated below the $x$ axis of each graph is shown. Line graphs show the percentage of cells possessing nuclear localized zyxin following treatment with IGF-1 $\left(\mathbf{A} ; 10^{-7} \mathrm{M}\right)$, adrenomedullin $\left(B ; 10^{-7} \mathrm{M}\right)$, and estradiol $\left(\mathbf{C} ; 1^{-7} \mathrm{M}\right)$ as assessed by confocal microscopy. Nuclear accumulation induced by any of the stimuli in $\mathbf{A}-\mathbf{C}$ was inhibited by pretreatment of the cultures with KT5823 (PKG inhibitor; $5 \times 10^{-6} \mathrm{M}$ ) as shown in D. $n=3$ for all experiments. ${ }^{\star} P<0.05$; ${ }^{*} P<0.01$. 

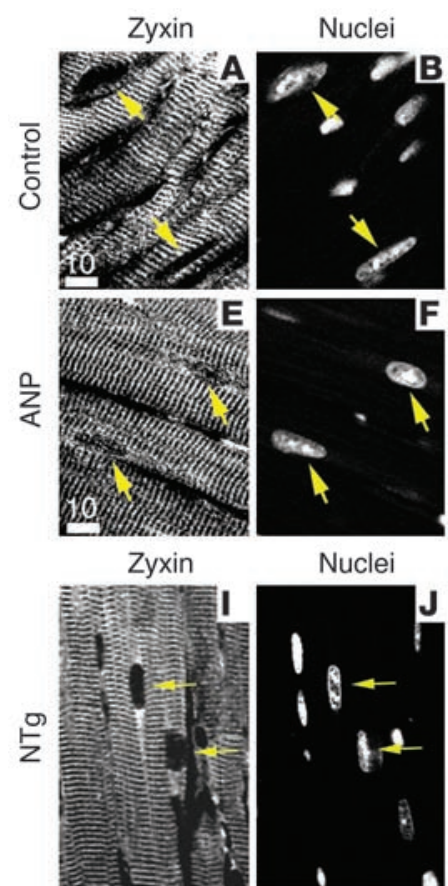

Nuclei
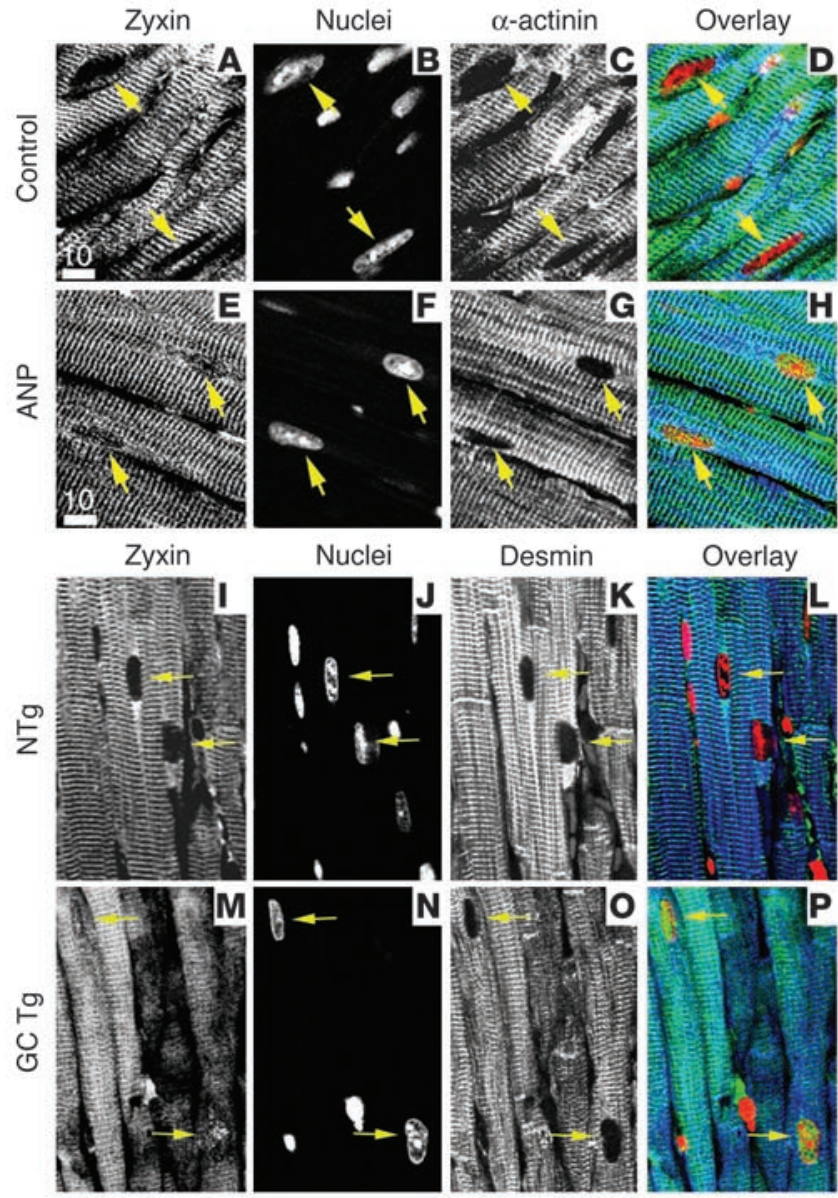

Desmin
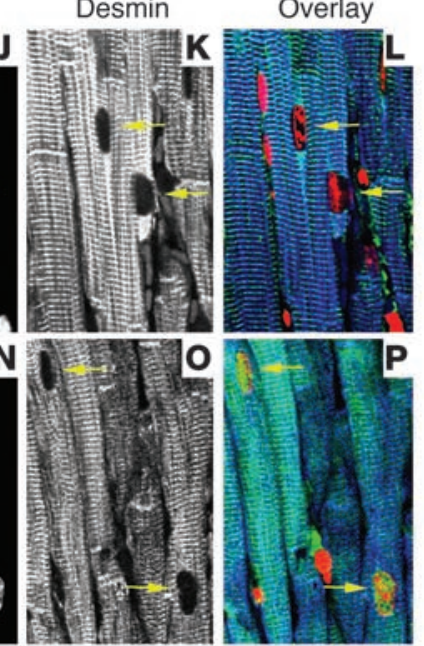

KK

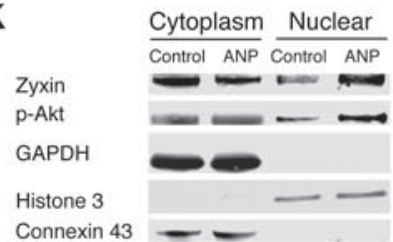

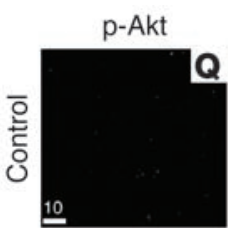
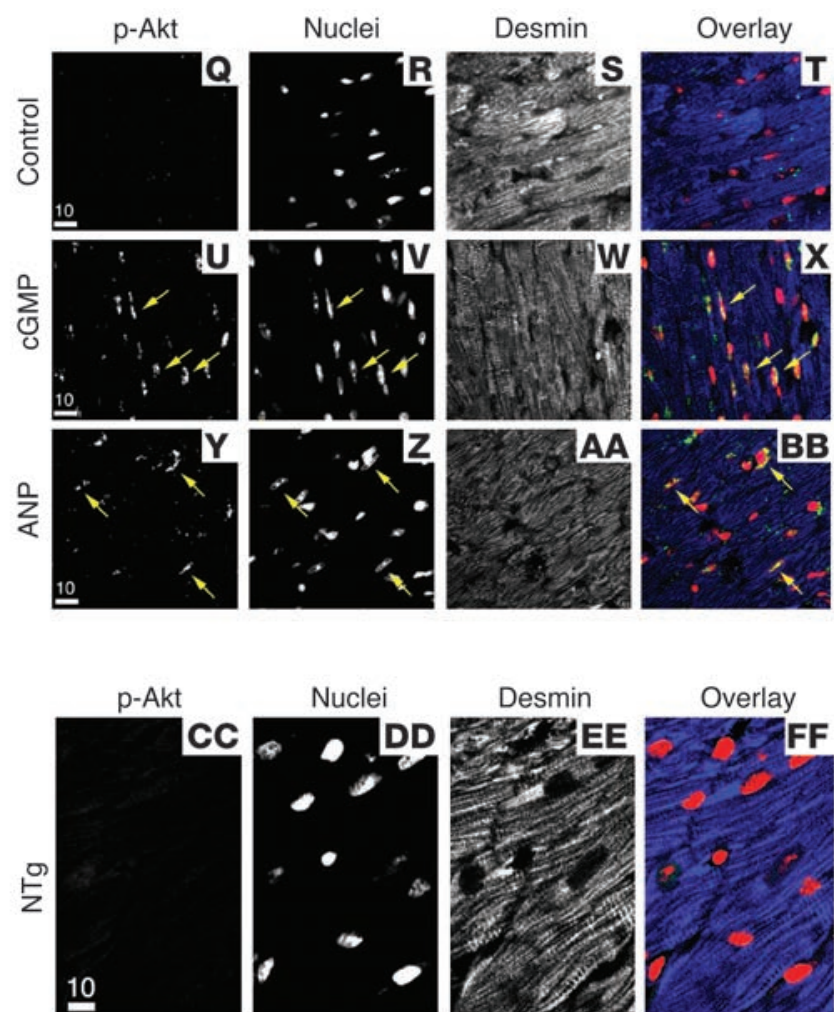

Desmin
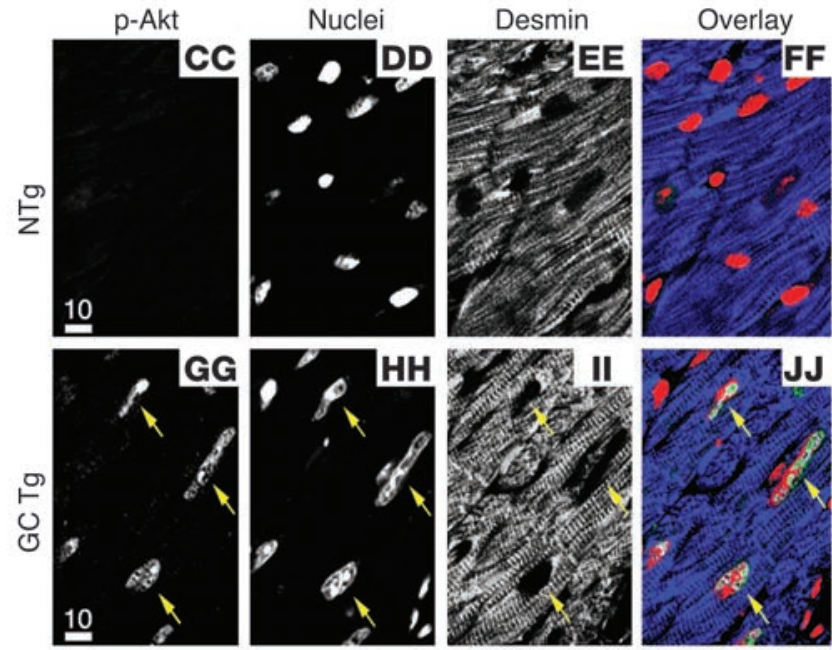

Figure 9

ANP induces nuclear accumulation of zyxin and phospho-Akt in the myocardium. Myocardial sections (A-JJ) and immunoblot (KK) demonstrating nuclear accumulation of zyxin or phospho-Akt 473 . All ANP treatments were performed for 24 hours using an implanted osmotic pump with $200 \mathrm{ul}$ of $10^{-4} \mathrm{M}$ ANP. For all cGMP treatments, agonist at a concentration of $10^{-2} \mathrm{M}$ was administered intravenously in $100 \mu \mathrm{l}$ volume, and hearts were removed 5 hours later. (A-P) Control (A-D and $\mathbf{I}-\mathbf{L})$ or experimental mice (E-H and $\mathbf{M}-\mathbf{P})$ labeled with antibody to zyxin (A, E, I, and $\mathbf{M}$; green in overlay). Cardiomyocytes were identified with antibodies to $\alpha$-actinin ( $\mathbf{C}$ and $\mathbf{G}$ ) or desmin (K and $\mathbf{O})$ (both blue in overlay). Nuclei were stained with TOPRO (B, F, J, and $\mathbf{N}$; red in overlay). Nuclear accumulation of zyxin was observed either following ANP treatment or in transgenic mice expressing cardiac-specific GC (GC Tg). (Q-JJ) Control (Q-T and CC-FF) or experimental (U-BB and GG-JJ) mouse hearts labeled with antibody to phospho-Akt ${ }^{473}$ (Q, $\mathbf{U}, \mathbf{Y}, \mathbf{C C}, \mathbf{G G}$; yellow in overlay in panels $\mathbf{X}, \mathbf{B B}$, and $\mathbf{J J}$ is due to coincident nuclear staining). Cardiomyocytes were identified with staining for desmin (S, W, AA, EE, and II; blue in overlays), and nuclei were identified by staining with TOPRO (R, V, Z, DD, and $\mathbf{H H}$; red in overlays). Nuclear accumulation of phospho-Akt 473 was observed following cGMP treatment (U-X; 5 hr intravenous), ANP treatment (Y-BB), andn in the cardiac-specific GC Tg mouse hearts (GG-JJ). (KK) Immunoblot of cardiac protein lysates from fractions of control or ANP-treated mice partitioned into nuclear and cytoplasmic/membrane fractions. Enrichment of zyxin or phospho-Akt ${ }^{473}$ was observed in samples from ANP-treated mice. Enrichment for nuclei was demonstrated by partitioning of GAPDH (cytoplasmic), histone H3 (nuclear), and connexin 43 (membrane associated) to appropriate fractions. Appropriate mobilities of bands on blots were confirmed using molecular weight standards in combination with labeling of unfractionated lysate (not shown). Scale bars: $10 \mu \mathrm{m}$.

or immunoprecipitates using an irrelevant control antibody, whereas zyxin immunoreactivity was evident in samples prepared from cardiomyocyte cultures treated with $10^{-9} \mathrm{M}$ ANP that increased within 1 hour after induction (Figure 10). Reciprocal immunoprecipitation experiments (IP for zyxin, then probe blot for Akt) yielded decreased signals for Akt (data not shown). Possible explanations for this finding include the following: (a) the zyxin antibodies available do not recognize the complex with Akt as efficiently as the noncomplexed form of the protein (epitope masking, conformational changes, etc.); (b) there may be 


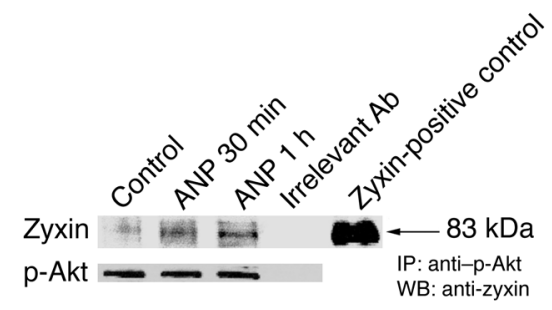

\section{Figure 10}

Cardioprotective stimuli induce association of activated Akt with zyxin. Lysates (300 mg total protein) prepared from cultured cardiomyocytes treated with vehicle (control) or ANP $\left(10^{-9} \mathrm{M}\right.$ for 30 or 60 minutes) were immunoprecipitated with anti-phospho-Akt 473 antibody, and presence of zyxin was detected by subsequent immunoblotting with anti-zyxin antibody. Whole cell lysate prior to immunoprecipitation is shown to indicate presence of zyxin (positive control). Immunolabeling with phospho-Akt ${ }^{473}$ antibody confirmed immunoprecipitation of protein (p-Akt). Immunoprecipitation with irrelevant antibody to anti-p16 ${ }^{\text {ink4a }}$ is shown as a negative control. WB, Western blot.

excess uncomplexed zyxin relative to activated Akt even in ANPstimulated cells; or (c) zyxin antibodies may not recognize native protein in solution as well as denatured protein on a blot.

Nuclear targeting of Akt induces nuclear accumulation of zyxin, and Akt activation is required for nuclear zyxin accumulation. Nuclear targeting of zyxin mediated nuclear accumulation of phospho$\mathrm{Akt}^{473}$ (Figure 4), and immunoprecipitation experiments suggested association between these 2 proteins (Figure 10). Thus, the capacity of Akt to serve in a reciprocal fashion to promote nuclear accumulation of zyxin was tested by adenoviral-mediated overexpression of Akt in wild-type or nuclear-targeted forms followed by confocal microscopy to determine zyxin distribution and the effect of Akt inhibition upon accumulation of zyxin in the nucleus. ANP-mediated nuclear accumulation of zyxin in cultured cardiomyocytes was blocked by inhibitors of Akt activity including LY294002, wortmannin, and adenoviral-mediated overexpression of dominant-negative Akt (Figure 11, A and B). Although pharmacologic inhibition by LY294002 or wortmannin effectively blocked zyxin nuclear accumulation (Figure 11B, blue bars), changes in zyxin distribution were evidenced by perinuclear (LY) or diffuse cytoplasmic (wortmannin) localization (Figure 11A). Adenoviral infection with various Akt constructs was performed to clarify the requirement for Akt activation in nuclear accumulation of zyxin. Nuclear accumulation of zyxin resulted from overexpression of wild-type Akt that is likely due to elevation of Akt activity, but overexpression of dominant-negative Akt did not increase nuclear zyxin accumulation (Figure 11B, green bars). Furthermore, dominant-negative Akt inhibited nuclear accumulation of zyxin mediated by ANP treatment (Figure 11B, red bars). Requirement for Akt activation is supported by overexpression of nuclear-targeted Akt, which loses the ability to promote nuclear accumulation of zyxin in the presence of LY294002 or wortmannin. Although nuclear-targeted Akt remained localized to the nucleus in the presence of the inhibitors, Akt activation as evaluated by immunoreactivity with antibodies to phospho$\mathrm{Akt}^{473}$ was lost (data not shown). Thus, localization of zyxin in the nucleus depends, in part, upon activation of Akt. Myocardial section from transgenic mice expressing nuclear-targeted Akt (32) also showed increased nuclear accumulation of zyxin compared with nontransgenic control samples (Figure 11, C-L, arrows), confirming the ability of Akt to promote nuclear accumulation of zyxin in the context of the myocardium.

\section{Discussion}

Natriuretic peptide administration exerts beneficial effects when used clinically for treatment of heart failure $(13,42)$ and in animal models to inhibit ischemic injury (43-45). Therapeutically, brain natriuretic peptide is infused at a rate of $0.01-0.03 \mu \mathrm{g} / \mathrm{kg} / \mathrm{min}$ versus ANP, which is infused at a concentration of $0.025 \mu \mathrm{g} / \mathrm{kg} / \mathrm{min}$, reaching plasma concentrations of $1.33 \mathrm{ng} / \mathrm{ml}(13)$ versus $1.6 \times 10^{-10} \mathrm{M}$ $(3,12)$, respectively. When used at levels close to physiological concentration $\left(10^{-11} \mathrm{M}\right)$, ANP stimulates cGMP-dependent protein kinase and Akt activation in human coronary arterial endothelial cells (46). Protective signaling induced by ANP could also account for suppression of serum deprivation-induced apoptosis in PC12 cells (14). In neural tumor cell lines, low concentrations $\left(10^{-10} \mathrm{M}\right)$ of ANP or C type natriuretic peptide stimulated proliferation, but higher concentrations exerted antiproliferative action (47). Consistent with our findings (Figure 1), natriuretic peptide concentration is a critical factor for influencing cell survival versus death, as apoptosis correlates with higher concentrations in dose-dependent exposure ranging from $10^{-9} \mathrm{M}$ to $10^{-6} \mathrm{M}(14-16,48)$. These results suggest that ANP exerts multiple independent actions upon target cells, including cardiomyocytes. A previous report linking ANP to promotion of cardiomyocyte apoptosis at $10^{-6}$ to $10^{-9} \mathrm{M}$ (48) never checked for short-term antiapoptotic actions of ANP and used serum-starved $(0 \%)$ cultures for assessment of apoptotic signaling. In our studies, serum starvation of myocyte cultures alone could promote apoptotic signaling, so the $2 \%$ serum concentration we used may have helped increase cell viability. Furthermore, we have observed in our system that apoptosis does occur in cardiac fibroblasts at the same low ANP concentrations that are protective for cardiomyocytes $\left(10^{-9}\right.$ to $10^{-10} \mathrm{M}$, not shown). Last, it should be noted that the detection methodology used by our group (TUNEL) is markedly different from the approach employed in the Wu et al. study (48), which assessed apoptosis by visualization of nuclear morphology with fluorescent DNA-binding dyes H33342 and propidium iodide. Potentiation of antiapoptotic signaling persisted after zyxin distribution has returned to the cytoskeleton (Figure 2C), so triggering of survival signaling involving zyxin translocation does not require long-term nuclear localization to maintain efficacy.

Nuclear accumulation of zyxin as a mediator of antiapoptotic signaling is an attractive candidate for participation in promotion of Akt activity based upon prior observations linking nuclear accumulation of Akt with resistance to apoptotic stimuli (32). Akt activation enhances myocardial cell survival in vitro (28) and in vivo (27) and accumulates in the nucleus in response to cardioprotective stimuli such as IGF-1 or estrogen (31), but mechanisms of nuclear trafficking remain obscure. Correlations between localization of zyxin and Akt in cells exposed to protective stimuli, observations indicating that presence of either protein in the nucleus promotes accumulation of its partner, and the induced coimmunoprecipitation of zyxin/Akt as a multimolecular complex following protective stimulation suggest interaction of these 2 proteins (although such interaction could certainly be indirectly brokered by additional as yet unidentified partners). At this point we do not know whether zyxin facilitates Akt transport to the nucleus or interacts with and helps retain Akt in the nucleus after arrival. Dependence of Akt transport or accumulation in the nucleus upon zyxin 
A Actin filaments
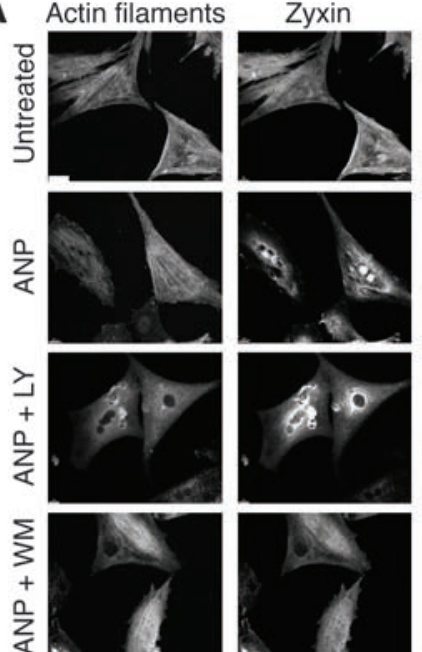

Nuclei
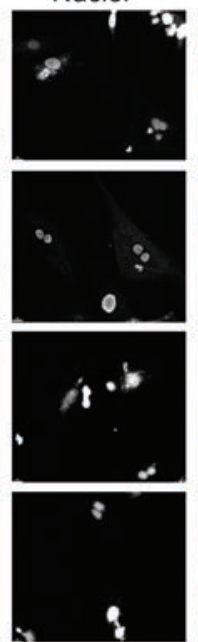

Overlay

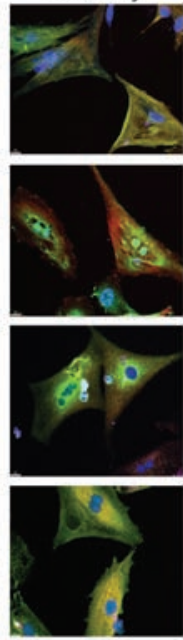

B

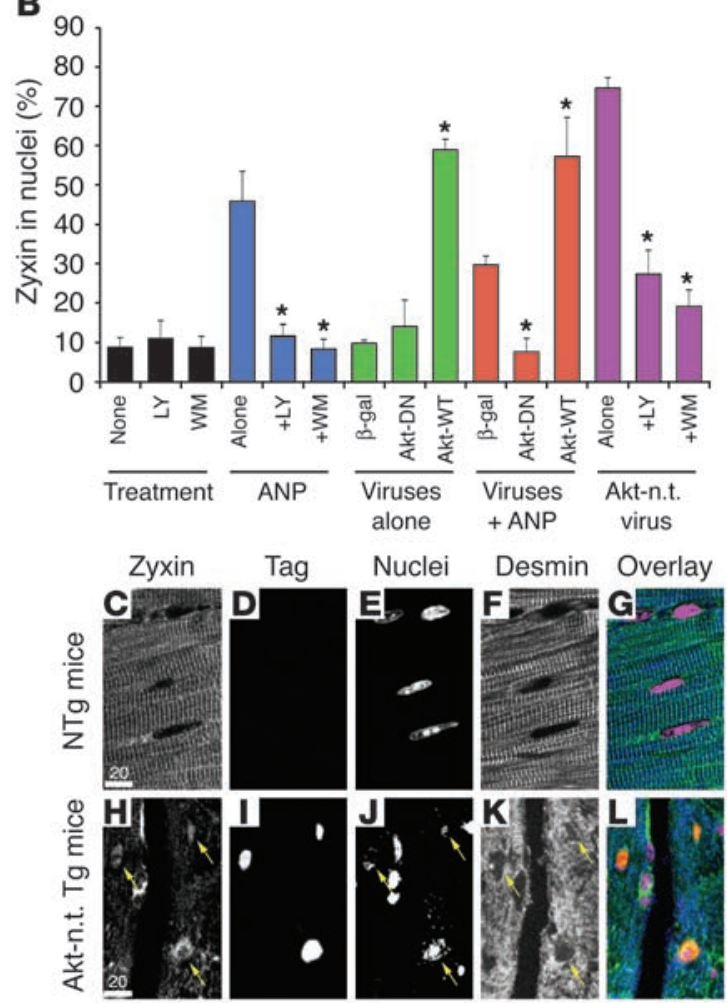

Figure 11

Nuclear-targeting of activated Akt induces nuclear accumulation of zyxin, and inhibition of Akt activation impairs nuclear accumulation of zyxin. Consequences of inhibiting Akt activation for zyxin nuclear localization (A and B) and Akt-mediated effects upon localization of zyxin in vivo (C-L). (A) Confocal micrographs of cultured cardiomyocytes treated with ANP alone or ANP following prior inhibitor treatments of LY294002 (LY) or wortmannin (WM). Distribution of zyxin (green in overlay) was influenced in all 3 groups, but inhibitor treatments prevented nuclear accumulation of zyxin. Nuclei were labeled with TOPRO (blue in overlay), and phalloidin shows actin filament structure (red). Scale bar: $10 \mu \mathrm{m}$ (all scans in A at identical scale). (B) Bar graph shows the percentage of cells possessing nuclear localized zyxin following treatment with ANP (10-9 M) as assessed by confocal microscopy. Treatment groups from left to right are: no treatment with pharmacologic inhibitors; ANP treatment with or without pharmacologic inhibitors; adenoviral infections without ANP treatment; adenoviral infections with ANP treatment; and nuclear-targeted Akt (Akt-n.t.) adenoviral infection with and without pharmacologic inhibitors. ${ }^{\star} P<0.005$ relative to the control (first bar of each group) was found as indicated. Myocardial sections (C-L) show nuclear accumulation of zyxin in transgenic mice expressing cardiac-specific nuclear-targeted Akt (Akt-n.t. Tg) but not in nontransgenic control samples. Arrows indicate nuclei positive for both zyxin and Akt-n.t. Transgenic Akt-n.t. was visualized using antibody to myc-tag (tag) and desmin to show myofibrillar structure. Arrows indicate nuclei positive for both Akt-n.t. and zyxin. Nuclei were shown to correlate with Akt-n.t. labeling but are not included in overlay to facilitate presentation. Overlay colors correspond to Akt-n.t. (red), zyxin (green), and desmin (blue) with coincidence of red and blue labeling appearing yellow. Scale bars: $20 \mu \mathrm{m}$ (C-L).

remains to be determined in future studies. Since zyxin exhibits a propensity for nuclear shuttling (35), it is tempting to postulate that the mechanism for nuclear accumulation of Akt in response to cardioprotective stimuli could involve zyxin. Characterization of the zyxin/Akt protein complex and identification of other possible members associated with these partners could provide valuable clues regarding molecular facilitators of nuclear trafficking and cell survival signaling (49). Since the carboxyl-LIM-domain protein family to which zyxin belongs consists of multiple members, it is probable that functional redundancy for mediating Akt nuclear accumulation exists, since zyxin knockout mice are overtly normal (50) whereas Akt knockout mice exhibit clear phenotypic abnormalities (51). Paxillin, a related carboxyl-LIM domain family member (52), exhibits striking similarities to zyxin in response to several of the parameters tested in our study (Supplemental Figure S3), suggesting the potential for functional redundancy between zyxin and paxillin within the limits of our analyses.
Cardioprotective stimuli and Akt activation have been linked via stimulation of cGMP production. Levels of cGMP rise following exposure to cardioprotective stimuli such as IGF-1 (40), adrenomedullin (38), estradiol (39), and natriuretic peptide, leading to models that demonstrate biological effect(s) of natriuretic peptides mediated by cGMP-dependent signaling (26). In a myocardial context, ANP inhibits hypertrophy of neonatal and adult cardiomyocytes through cGMP-dependent signaling involving $\mathrm{pGC}$ receptors $(53,54)$. Similar to dose-dependent effects of ANP upon cell survival, cGMP can promote $(55,56)$ or inhibit $(57)$ apoptotic cell death. ANP-mediated effects were mimicked by manipulation of cGMP signaling (Figures 5-7) that together with PKG involvement (Figure 5) adds another piece to the emerging picture of the ANP-dependent survival signaling cascade.

Nuclear accumulation of zyxin in vivo is induced by circulating ANP (Figure 9), indicating this cardioprotective autocrine/paracrine signaling mechanism operates in the myocardium. The short 
half life of ANP as measured in humans (10-20 minutes; ref. 58) or rats (a few minutes; ref. 59) prompted use of an osmotic pump for prolonged release (Figure 9, I-P). Clinically, our results are consistent with elevation of plasma cGMP following intravenous infusion of ANP (12) and the beneficial effects of left ventricular assist device support leading to normalization of natriuretic peptide levels and recovery of GC-A activity (60). The in vivo situation is complex and likely involves concurrent effects of GCs stimulated by activation of nitric oxide production (61).

Natriuretic peptide receptor desensitization occurs in congestive heart failure (CHF) (62). Among patients with mild CHF, the plasma cGMP level correlated with the ANP level. In contrast, these correlations were not found in patients with severe CHF. This suggests that downregulation of natriuretic peptide receptor in CHF could indeed attenuate ANP-mediated effects due to lack of cGMP induction. Since mice in our study were not in heart failure, these issues are not a factor, but it is clearly important to point out this limitation when interpreting our results in the context of therapeutic effects of exogenously administered natriuretic peptides.

The paradigm of signal transduction elucidated in this study involving the nuclear accumulation of zyxin and Akt provides directions for future studies to unify the intracellular trafficking of cytoskeletal LIM proteins to cardioprotection. Future studies will expand upon the relationship of LIM-domain proteins to cardioprotective stimulation and cGMP-mediated signaling, leading to better understanding of cardiac pathogenesis and novel therapeutic approaches for molecular intervention in the pathogenesis of heart failure.

\section{Methods}

Cardiomyocyte preparation. Neonatal rat ventricular myocytes were isolated from hearts of 2- to 3-day-old Sprague-Dawley rats by multiple rounds of digestion using a combination of collagenase type I (Worthington) and pancreatin as previously described (31). Cells prepared in this way were preplated for 2 hours in M-199 plus 15\% FBS to reduce nonmyocyte contamination and plated at various densities on either plastic chamber slides pretreated with laminin (Sigma-Aldrich) or $1 \%$ gelatin-coated tissue culture dishes. On the following day, myocyte cultures were washed and refed with maintenance medium (M-199 supplemented with $2 \%$ horse serum) prior to incubation for up to 3 days. Bromodeoxyuridine was used to inhibit fibroblast proliferation, and $98-99 \%$ purity of our cultures for cardiomyocytes was confirmed by direct observation with phalloidin labeling.

Adenoviruses. Adenoviruses for pyk2/RAFTK, GFP, $\beta$-gal, and nucleartargeted Akt were prepared as previously described $(31,32,37)$. GFPtagged nuclear-targeted zyxin was cloned using overlap PCR as described (63). Nuclear-targeted zyxin adenovirus was created with external primers generated complementary to the $5^{\prime}$ end of enhanced GFP (EGFP) (Clontech) with a $5^{\prime}$ NheI site (P1) and the $3^{\prime}$ end of the myc tag and the nuclear localization sequence from $\mathrm{pCMV} / \mathrm{myc} /$ nuc (Invitrogen) with a stop codon in frame and SalI at $3^{\prime}$ terminus (P4AS). Internal primers were generated with $18 \mathrm{bp}$ overlaps (36 mer total) into the upstream and downstream coding sequences of the genes to be fused. The primers (P2S and P2AS) corresponding to the fusion region between the $3^{\prime}$ end of EGFP and the $5^{\prime}$ end of murine zyxin contained $18 \mathrm{bp}$ of each sequence for a total length of $36 \mathrm{bp}$, as did the primers (P3S and P3AS) corresponding to the fusion region between the $3^{\prime}$ end of murine zyxin and the $5^{\prime}$ end of the nuclear localization sequence (NLS) concatamer. PCR was then performed on each fragment using the corresponding primer sets with Hi-Fidelity Supermix (Invitrogen Corp.), and PCR fragments were gel-purified. Fragments were then combined in equimolar ratios in Hi-Fidelity Supermix (Invitrogen Corp.), and fusion PCR was performed. The complete fusion gene was then ligated into PCRITOPO using Invitrogen's TOPO-TA cloning kit. Colonies were analyzed by PCR, and positive colonies were grown, miniprepped, and sent for complete sequencing. A plasmid containing the verified sequence for the EGFPZyxin-NLS cDNA was digested with NheI and SalI, and the resultant $2.5-\mathrm{kb}$ GFP-Zyxin-NLS fragment was gel isolated and ligated into pDC315io (Microbix). Resultant colonies were analyzed by PCR, miniprepped, and confirmed by sequencing. The plasmid with the verified sequence was subsequently cotransfected in an equimolar ratio with pBHGlox $\Delta \mathrm{E} 1,3 \mathrm{Cre}$ (Microbix) using PolyFect transfection reagent (QIAGEN) into passage 14293 iq cells from Microbix to generate the adenovirus. Appropriate expression of the protein of interest was confirmed by immunoblot and immunohistochemistry of infected cardiomyocytes.

Transgenic mouse lines. All animal procedures were approved by the Institutional Animal Care and Use Committee of San Diego State University. Transgenic mice expressing cardiac-specific nuclear-targeted Akt were previously described (32), and mice expressing cardiac-specific constitutively active GC (41) were generously provided by Christian Deschepper (Institut de Recherches Cliniques, Montreal, Quebec, Canada). Nuclear-targeted zyxin transgenic mice were generated using the pDCGFP-Zyxin-NLS plasmid digested with NheI/SalI, and the resulting 2.5-kb GFP-ZyxinNLS fragment was gel purified. The fragment was subsequently ligated downstream of the NheI/XhoI-digested $\alpha \mathrm{MHC}$ promoter plasmid (kindly provided by Jeffrey Robbins, Children's Hospital Medical Center, Cincinnati, Ohio, USA). Colony PCR was performed, and positive plasmids were miniprepped and sent for sequencing. The c26L.5GFP-Zyxin-NLS.14 sequenced plasmid was subsequently digested with BamHI to release the plasmid backbone, and the $8.5 \mathrm{~kb}$ promoter and cDNA gel purified and microinjected using established protocols into $0.5 \mathrm{dpc}$ (days post coitum) FVBN/J embryos. Resulting founders were analyzed by PCR using primers $\alpha$ MHC (-550): GAATCACACCTGGGGTTCCC and EGFP $(+140)$ : TCAGGGTCAGCTTGCCGTAG and cycling conditions of $95^{\circ} \mathrm{C}$ for 3 minutes, 33 cycles of $95^{\circ} \mathrm{C}$ for 30 seconds, $57^{\circ} \mathrm{C}$ for 30 seconds, $72^{\circ} \mathrm{C}$ for 1 minute, and a final hold at $72^{\circ} \mathrm{C}$ for 10 minutes resulting in a 703 -bp product. Positive founders were backcrossed to FVBN/J to produce nuclear-targeted zyxin mice used in the experiments.

Immunoblotting. Whole cell lysates were prepared from cultured cells by first washing cardiomyocytes twice with PBS, then directly adding cell culture lysis buffer as previously described (64). Tissue lysates from cardiac tissue were prepared from hearts collected from 6- to 8-week-old mice and homogenized in tissue lysis buffer as previously described (65). Enrichment for nuclear fractions of samples was performed using the NE-PER kit as recommended by the manufacturer (Pierce Biotechnology Inc.). Extracts were briefly sonicated and microfuged 10 minutes prior to electrophoresis. Fifty $\mu \mathrm{g}$ per lane of protein was mixed with an equal volume of sample buffer and boiled for 5 minutes before being loaded onto a $10 \%$ SDS-PAGE gel for electrophoresis. Separated proteins were transferred to Hybond transfer membranes (Amersham Pharmacia Biotech) using a Trans-Blot Cell (Bio-Rad Laboratories) at $145 \mathrm{~V}$ for 3 hours at $4{ }^{\circ} \mathrm{C}$. Blots were rinsed in water, washed in TBST $(50 \mathrm{mmol} / 1$ Tris$\mathrm{Hcl} \mathrm{pH} \mathrm{7.6,} 150 \mathrm{mM} \mathrm{NaCl}$, and $0.1 \%$ Tween 20) and incubated 1 hour in blocking solution (5\% BSA in TBST). Blots were incubated overnight at $4^{\circ} \mathrm{C}$ in primary antibodies against either zyxin (Sigma-Aldrich) or cleaved caspase-3 (Cell Signaling Technology) dissolved in blocking solution. The next day, membranes were washed 3 times, 5 minutes each, in TBST prior to a 1 -hour incubation at $25^{\circ} \mathrm{C}$ with fluorescent-conjugated secondary antibody (Jackson Laboratory) dissolved in blocking solution. 
Membranes were washed and reactive proteins visualized by fluorescence using a Typhoon 9410 scanner (GE Healthcare). Quantitation of zyxin and cleaved caspase- 3 protein content was performed using ImageQuant 1.2 software provided with the Typhoon scanner. Histone H3 (Cell Signaling Technology) immunolabeling was used to standardize protein loading among nuclear extracts. Partitioning of the membrane fraction was verified using antibody to connexin 43 (Zymed Laboratories Inc.).

Immunoprecipitation protocol. Neonatal cardiomyocytes were grown in $150-\mathrm{mm}$ plates to confluence. Cells were scraped and lysed in $400 \mu \mathrm{l}$ of immunoprecipitation buffer on ice following sonication for 1 second 8 times. The immunoprecipitation buffer had the same constitutents as lysis buffer for immunoblot protocols. Antibodies to phospho-Akt ${ }^{473}$ (BioSource International), zyxin (Santa Cruz Biotechnology Inc.), paxillin (BD Transduction Laboratories; BD Biosciences - Pharmingen), or PI3-K (Upstate) were added at a dilution of 1:100 in a total volume of $500 \mu \mathrm{l}$, and immunoprecipitation was performed at $4{ }^{\circ} \mathrm{C}$ overnight with constant mixing. Protein $\mathrm{G}$ beads $(25 \mu \mathrm{l})$ were added, and the solution was allowed to mix for 3 hours at $4^{\circ} \mathrm{C}$. Protein $\mathrm{G}$ beads were washed 3 times using the immunoprecipitation buffer and then prepared for PAGE by suspension in PAGE sample buffer.

Confocal microscopy. Cardiomyocytes cultured in 2-well chamber slides were rinsed in PBS and fixed in $4 \%$ paraformaldehyde. Cells were subsequently rehydrated in PBS for 10 minutes, washed in PBS containing $0.1 \mathrm{mM}$ glycine for 5 minutes, rinsed briefly in PBS, permeabilized in PBS containing $0.5 \%$ Triton X-100, washed twice in 3-minute PBS rinses, and blocked for 1 hour in PBS containing 10\% horse serum. Slides were incubated overnight at $4{ }^{\circ} \mathrm{C}$ with primary antibodies diluted in blocking solution. The next day, slides were washed 3 times for 10 minutes each time in PBS and incubated for 1 hour at room temperature in the dark, with secondary antibodies conjugated to either FITC or Texas Red diluted 1:100 in blocking solution (Jackson ImmunoResearch Laboratories Inc.). After secondary labeling, slides were washed 3 times in PBS for 5 minutes per wash and mounted for viewing in VECTASHIELD medium (Vector Laboratories). Confocal images were acquired using a Molecular Dynamics CLSM 2010 as previously described (31). For identification of zyxin, rabbit anti-zyxin (Sigma-Aldrich) was used as a primary $\mathrm{Ab}$ and was followed by FITC-conjugated donkey anti-rabbit IgG (Jackson ImmunoResearch Laboratories). Desmin was detected by goat anti-desmin (Santa Cruz Biotechnology Inc.) followed by Texas Red-conjugated donkey anti-goat IgG (Jackson ImmunoResearch Laboratories). Rabbit anti-phospho-Akt ${ }^{473}$ (BioSource International) was used as a primary antibody to identify phospho-Akt ${ }^{473}$, followed by FITC-conjugated donkey anti-rabbit IgG (Jackson ImmunoResearch Laboratories). The myctag of nuclear-targeted Akt was detected by mouse anti-c-myc (Santa Cruz Biotechnology Inc.) followed by Texas Red-conjugated donkey anti-mouse IgG (Jackson ImmunoResearch Laboratories). Paxillin was detected by anti-paxillin antibody (BD Transduction Laboratories; BD Biosciences - Pharmingen). TOPRO-3 (Invitrogen Corp.) staining was performed to determine the localization of nuclei.

In vitro treatments. Estradiol, adrenomedullin, IGF-1, PMA, forskolin, KT5823, and atrial natriuretic peptide were purchased from SigmaAldrich. HS142-1, a natriuretic peptide receptor A- and B-specific inhibitor, was a generous gift from H. Yano (Kyowa Hakko Kogyo Co., Tokyo, Japan). Cultures were treated with KT5823 or HS-142 1 hour prior to ANP stimulation. The cGMP analog used was cell permeable 8 bromoguanosine 3', 5'-cyclic monophosphate sodium salt monohydrate (B1893; Sigma-Aldrich).

In vivo treatments. Subcutaneously implanted osmotic pumps $(200 \mu \mathrm{l}$, ALZET) containing ANP $\left(10^{-4} \mathrm{M}\right)$ were employed for 24 hours. Intravenous infusion of saline and ANP was done through the tail vein at a rate of approximately $50 \mu \mathrm{l} / \mathrm{min}$. Infusion volumes were finally adjusted to $100 \mu \mathrm{l}$ before administration.

Histological analyses. Sections were prepared from hearts that were fixed for 24 hours in neutral buffered formalin at room temperature. Paraffin-embedded tissue sections were prepared from mouse hearts that had been fixed in formalin overnight at $4^{\circ} \mathrm{C}$. The next day, hearts were infiltrated with paraffin by an automated tissue processor. Paraffin-embedded hearts were sectioned at a thickness of $5 \mu \mathrm{m}$ followed by deparaffinization and rehydration.

Apoptosis determination assays. For TUNEL assays, cells were fixed and then labeled using terminal deoxyribonucleotidyl transferase according to the manufacturer's instructions (In Situ Apoptosis Detection Kit; Roche Diagnostics Corp.). In the colorimetric assay, apoptotic cells appeared as bright orange. Quantitative colocalization analysis for TUNEL positive nuclei was performed using CoLocalizer Pro version 1.2 (CoLocalization Research Software). DNA laddering was analyzed as described (66). DNA fragments ( $20 \mu \mathrm{g}$ each) were separated by $1.2 \%$ agarose gel electrophoresis.

Statistics. Results are expressed as means \pm SEM. Differences in quantitative variables were examined by 1-way ANOVA and Tukey post hoc test for multiple comparisons.

ANP ELISA. ELISAs for ANP were performed using the ANF Rat (1-28) Immunoassay kit (89995; Peninsula Laboratories). Serum samples were drawn on anesthetized mice via cardiac puncture into syringes containing $2.7 \mathrm{mM}$ EDTA, $600 \mu \mathrm{M}$ PMSF, and $200 \mathrm{KIU}$ aprotinin, and centrifuged at $16,000 \mathrm{~g}, 4^{\circ} \mathrm{C}$ for 15 minutes. Isolated serum was extracted over a Sep-Pak C-18 cartridge (Y1000; Peninsula Laboratories) per kit instructions, resuspended in ELISA buffer, and analyzed.

Ex vivo ischemia/reperfusion and hemodynamic analyses. Hearts were excised and treated in a Langendorff model system as previously described (67). In brief, hearts were removed and placed in chilled Krebs buffer for aortic cannulation. Perfused hearts were mounted on the Langendorff system at $80 \mathrm{~mm} \mathrm{Hg}$ aortic pressure, and a balloon was placed into the left ventricle on a closed system containing a Millar transducer. Hearts were perfused for a 30-minute equilibration period followed by 30 minutes of no-flow ischemia, after which fluid flow was resumed for a reperfusion time of 2 hours followed by 5 -minute perfusion with neutral buffered formalin. Pressure measurements were recorded using an ADInstruments PowerLab system. Data was graphed as the percentage of preischemic left ventricular developed pressure. Five mice were used for each group except for the NT overexpressors where 4 animals were studied. ANOVA was done using SPSS software version 11.0 (SPSS Inc.) and a Newman-Keuls post hoc analysis.

Inbibitor experiments. Neonatal cardiomyocytes were infected with adenoviruses for $\beta$-gal, dominant-negative Akt (kindly provided by Thomas Franke, Columbia University, New York, New York, USA), nuclear-targeted Akt, and wild-type Akt for 2 hours. Following infection, cultured cardiomyocytes plated on chamber slides were refed with M199 and 2\% FBS. Cells that were not infected were serum starved in M199 and 2\% FBS for 24 hours prior to treatments. After 24 hours, cells were treated with $50 \mu \mathrm{M}$ LY294002 or $100 \mathrm{nM}$ wortmannin (both purchased from SigmaAldrich) for 1 hour prior to the 1-hour ANP $\left(10^{-9} \mathrm{M}\right)$ treatment. Following ANP treatment, cells were prepared for immunofluorescence microscopy. The number of cells with nuclear localized zyxin were counted on a fluorescent scope for each treatment condition and slide.

\section{Acknowledgments}

This work was supported by NIH grants HL58224, HL66035, and HL67245 to M. Sussman as well as Component 4 of Program Project grant PO1 AG023071 (Piero Anversa, Director). M. Beckerle is supported by NIH grant GM50877, and C. Glembotski is 
supported by NIH grants HL075575 and NS/HL 025037. T. Kato was supported by a grant from the Uehara Memorial Foundation. J. Muraski is a Fellow of the Rees-Stealy Research Foundation and the San Diego State University Heart Institute. We also thank M. Kato (Edogawa Hospital), J. Tomioka (Edogawa Hospital), H. Shigemune (Kyowa Hakko Kogyo Co.), H. Yano (Kyowa Hakko Kogyo Co.), Natalie Gude, and Leslie Rodelander for invaluable assistance.
Received for publication December 21, 2004, and accepted in revised form July 19, 2005.

Address correspondence to: Mark A. Sussman, San Diego State University, SDSU Heart Institute and Department of Biology, Room 426, 5500 Campanile Drive, San Diego, California 92182, USA. Phone: (619) 594-2983; Fax: (619) 594-2610; E-mail: sussman@heart.sdsu.edu.
1. Pidgeon, G.B., et al. 1996. Differing metabolism and bioactivity of atrial and brain natriuretic peptides in essential hypertension. Hypertension. 27:906-913.

2. Tonolo, G., et al. 1989. Low-dose infusion of atrial natriuretic factor in mild essential hypertension. Circulation. 80:893-902.

3. Hayashi, M., et al. 2001. Intravenous atrial natriuretic peptide prevents left ventricular remodeling in patients with first anterior acute myocardial infarction. J. Am. Coll. Cardiol. 37:1820-1826.

4. Lai, C.P., et al. 1993. Beneficial effects of atrial natriuretic peptide on exercise-induced myocardial ischemia in patients with stable effort angina pectoris. Circulation. 87:144-151.

5. Rosenthal, A.D., Moran, M., and Herrmann, H.C. 1990. Coronary hemodynamic effects of atrial natriuretic peptide in humans. J. Am. Coll. Cardiol. 16:1107-1113.

6. Herrmann, H.C., Rosenthal, A.D., and Davis, C.A. 1990. Cardiovascular effects of intracoronary atrial natriuretic peptide administration in man. Am. Heart J. 120:308-315.

7. Kato, H., et al. 1994. Suppression of hyperventilation-induced attacks with infusion of B-type (brain) natriuretic peptide in patients with variant angina. Am. Heart J. 128:1098-1104.

8. Clarkson, P.B., et al. 1996. Effects of brain natriuretic peptide on exercise hemodynamics and neurohormones in isolated diastolic heart failure. Circulation. 93:2037-2042.

9. Molina, C.R., et al. 1988. Hemodynamic, renal and endocrine effects of atrial natriuretic peptide infusion in severe heart failure. J. Am. Coll. Cardiol. 12: $175-186$

10. Fifer, M.A., et al. 1990. Hemodynamic and renal effects of atrial natriuretic peptide in congestive heart failure. Am. J. Cardiol. 65:211-216.

11. Nakamura, M., et al. 1998. Vasodilatory effects of B-type natriuretic peptide are impaired in patients with chronic heart failure. Am. Heart J. 135:414-420.

12. Mizuno, O., Onishi, K., Dohi, K., and Nakano, T. 2001. Effects of therapeutic doses of human atrial natriuretic peptide on load and myocardial performance in patients with congestive heart failure. Am. J. Cardiol. 88:863-866.

13. Colucci, W.S., et al. 2000. Intravenous nesiritide, a natriuretic peptide, in the treatment of decompensated congestive heart failure. Nesiritide Study Group. N. Engl. J. Med. 343:246-253.

14. Fiscus, R.R., Tu, A.W., and Chew, S.B. 2001. Natriuretic peptides inhibit apoptosis and prolong the survival of serum-deprived PC12 cells. Neuroreport. 12:185-189.

15. Matsumura, T., et al. 1996. Neutral endopeptidase 24.11 in neutrophils modulates protective effects of natriuretic peptides against neutrophils-induced endothelial cytotoxity. J. Clin. Invest. 97:2192-2203.

16. Cottart, C.H., et al. 2003. Hepatic cytoprotection by nitric oxide and the cGMP pathway after ischemia-reperfusion in the rat. Nitric Oxide. 9:57-63.

17. Suenobu, N., Shichiri, M., Iwashina, M., Marumo, F., and Hirata, Y. 1999. Natriuretic peptides and nitric oxide induce endothelial apoptosis via a cGMP-dependent mechanism. Arterioscler. Thromb. Vasc. Biol. 19:140-146.
18. Han, B., et al. 2003. The opposing effects of endothelin-1 and C-type natriuretic peptide on apoptosis of neonatal rat cardiac myocytes. Eur. J. Pharmacol. 474:15-20.

19. Kato, K., et al. 2003. Adrenomedullin gene delivery attenuates myocardial infarction and apoptosis after ischemia and reperfusion. Am.J. Physiol. Heart Circ. Physiol. 285:H1506-H1514.

20. Wollert, K.C., et al. 2002. Gene transfer of cGMPdependent protein kinase I enhances the antihypertrophic effects of nitric oxide in cardiomyocytes. Hypertension. 39:87-92.

21. Begum, N., Sandu, O.A., Ito, M., Lohmann, S.M., and Smolenski, A. 2002. Active Rho kinase (ROKalpha) associates with insulin receptor substrate-1 and inhibits insulin signaling in vascular smooth muscle cells. J. Biol. Chem. 277:6214-6222.

22. Hood, J., and Granger, H.J. 1998. Protein kinase $G$ mediates vascular endothelial growth factorinduced Raf- 1 activation and proliferation in human endothelial cells. J. Biol. Chem. 273:23504-23508.

23. Kawasaki, K., et al. 2003. Activation of the phosphatidylinositol 3-kinase/protein kinase Akt pathway mediates nitric oxide-induced endothelial cell migration and angiogenesis. Mol. Cell. Biol. 23:5726-5737.

24. Chang, M.S., Lee, W.S., Chen, B.C., Sheu, J.R., and Lin, C.H. 2004. YC-1-induced cyclooxygenase-2 expression is mediated by cGMP-dependent activations of Ras, phosphoinositide-3-OH-kinase, Akt, and nuclear factor-kappaB in human pulmonary epithelial cells. Mol. Pharmacol. 66:561-571.

25. Pollman, M.J., Yamada, T., Horiuchi, M., and Gibbons, G.H. 1996. Vasoactive substances regulate vascular smooth muscle cell apoptosis: countervailing influences of nitric oxide and angiotensin II. Circ. Res. 79:748-756.

26. Fiscus, R.R. 2002. Involvement of cyclic GMP and protein kinase $\mathrm{G}$ in the regulation of apoptosis and survival in neural cells. Neurosignals. 11:175-190.

27. Fujio, Y., Nguyen, T., Wencker, D., Kitsis, R.N., and Walsh, K. 2000. Akt promotes survival of cardiomyocytes in vitro and protects against ischemiareperfusion injury in mouse heart. Circulation. 101:660-667.

28. Matsui, T., Li, L., and Rosenzweig, A. 1999. Adenoviral gene transfer of activated phosphatidylinositol 3'-kinase and Akt inhibits apoptosis of hypoxic cardiomyocytes in vitro. Circulation. 100:2373-2379.

29. Negoro, S., et al. 2001. Glycoprotein 130 regulates cardiac myocyte survival in doxorubicin-induced apoptosis through phosphatidylinositol 3-kinase/Akt phosphorylation and $\mathrm{Bcl}-\mathrm{xL} / \mathrm{caspase}-3$ interaction. Circulation. 103:555-561.

30. Torella, D., et al. 2004. Cardiac stem cell and myocyte aging, heart failure, and insulin-like growth factor-1 overexpression. Circ. Res. 94:514-524.

31. Camper-Kirby, D., et al. 2001. Myocardial Akt activation and gender: increased nuclear activity in females versus males. Circ. Res. 88:1020-1027.

32. Shiraishi, I., et al. 2004. Nuclear targeting of Akt enhances kinase activity and survival of cardiomyocytes. Circ. Res. 94:884-891.

33. Cattaruzza, M., Lattrich, C., and Hecker, M. 2004. Focal adhesion protein zyxin is a mechanosensitive modulator of gene expression in vascular smooth muscle cells. Hypertension. 43:726-730.

34. Smolenski, A., Poller, W., Walter, U., and Lohmann, S.M. 2000. Regulation of human endothelial cell focal adhesion sites and migration by cGMP-dependent protein kinase I. J. Biol. Chem. 275:25723-25732.

35. Nix, D.A., and Beckerle, M.C. 1997. Nuclear-cytoplasmic shuttling of the focal contact protein, zyxin: a potential mechanism for communication between sites of cell adhesion and the nucleus. J. Cell Biol. 138:1139-1147.

36. Nix, D.A., et al. 2001. Targeting of zyxin to sites of actin membrane interaction and to the nucleus. J. Biol. Chem. 276:34759-34767.

37. Melendez, J., et al. 2004. Cardiomyocyte apoptosis triggered by RAFTK/pyk 2 via src kinase is antagonized by paxillin. J. Biol. Chem. 279:53516-53523.

38. Yin, H., Chao, L., and Chao, J. 2004. Adrenomedullin protects against myocardial apoptosis after ischemia/reperfusion through activation of AktGSK signaling. Hypertension. 43:109-116.

39. Babiker, FA., et al. 2004. 17beta-estradiol antagonizes cardiomyocyte hypertrophy by autocrine/ paracrine stimulation of a guanylyl cyclase A receptor-cyclic guanosine monophosphate-dependent protein kinase pathway. Circulation. 109:269-276.

40. Bonthius, D.J., Karacay, B., and Pantazis, N.J. 2003. FGF-2, NGF and IGF-1, but not BDNF, utilize a nitric oxide pathway to signal neurotrophic and neuroprotective effects against alcohol toxicity in cerebellar granule cell cultures. Brain Res. Dev. Brain Res. 140:15-28.

41. Zahabi, A., Picard, S., Fortin, N., Reudelhuber, T.L., and Deschepper, C.F. 2003. Expression of constitutively active guanylate cyclase in cardiomyocytes inhibits the hypertrophic effects of isoproterenol and aortic constriction on mouse hearts. J. Biol. Chem. 278:47694-47699.

42. Publication Committee for the VMAC Investigators (Vasodilatation in the Management of Acute CHF). 2002. Intravenous nesiritide vs nitroglycerin for treatment of decompensated congestive heart failure: a randomized controlled trial. JAMA. 287:1531-1540.

43. D'Souza, S.P., et al. 2003. B-type natriuretic peptide limits infarct size in rat isolated hearts via KATP channel opening. Am. J. Physiol. Heart Circ. Physiol. 284:H1592-H1600.

44. Kamijo, T., et al. 1999. Hemodynamic and hormonal responses to nicorandil in a canine model of acute ischemic heart failure: a comparison with cromakalim and nitroglycerin. J. Cardiovasc. Pharmacol. 33:93-101.

45. Padilla, F., Garcia-Dorado, D., Agullo, L., Pina, P., and Soler-Soler, J. 2001. Intravenous administration of the natriuretic peptide urodilatin at low doses during coronary reperfusion limits infarct size in anesthetized pigs. Cardiovasc. Res. 51:592-600.

46. Kook, H., Itoh, H., and Nakao, K. 2003. Physiological concentration of atrial natriuretic peptide induces endothelial regeneration in vitro. Am. J. Physiol. Heart Circ. Physiol. 284:H1388-H1397.

47. Lelievre, V., Pineau, N., Hu, Z., and Waschek, J.A. 2001. Proliferative actions of natriuretic peptides on neuroblastoma cells. Involvement of guanylyl cyclase and non-guanylyl cyclase pathways. J. Biol. Chem. 276:43668-43676. 
48. Wu, C., Bishopric, N.H., and Pratt, R.E. 1997. Atrial natriuretic peptide induces apoptosis in neonatal rat cardiac myocytes. J. Biol. Chem. 272:14860-14866

49. Kadrmas, J.L., and Beckerle, M.C. 2004. The LIM domain: from the cytoskeleton to the nucleus. Nat. Rev. Mol. Cell Biol. 5:920-931.

50. Hoffman, L.M., et al. 2003. Targeted disruption of the murine zyxin gene. Mol. Cell. Biol. 23:70-79.

51. Cho, H., Thorvaldsen, J.L., Chu, Q., Feng, F., and Birnbaum, M.J. 2001. Akt1/PKBalpha is required for normal growth but dispensable for maintenance of glucose homeostasis in mice. J. Biol. Chem. 276:38349-38352.

52. Brown, M.C., and Turner, C.E. 2004. Paxillin: adapting to change. Physiol. Rev. 84:1315-1339.

53. Calderone, A., Thaik, C., Takahashi, N., Chang, D.L.F., and Colucci, W.S. 1998. Nitric oxide, atrial natriuretic peptide and cyclic GMP inhibit the growth-promoting effects of norepinephrine in cardiac myocytes and fibroblasts. J. Clin. Invest. 101:812-818.

54. Rosenkranz, A.C., Woods, R.L., Dusting, G.J., and Ritchie, R.H. 2003. Antihypertrophic actions of the natriuretic peptides in adult rat cardiomyocytes: importance of cyclic GMP. Cardiovasc. Res. 57:515-522.
55. Shimojo, T., et al. 1999. Nitric oxide induces apoptotic death of cardiomyocytes via a cyclic-GMPdependent pathway. Exp. Cell Res. 247:38-47.

56. Arstall, M.A., Sawyer, D.B., Fukazawa, R., and Kelly, R.A. 1999. Cytokine-mediated apoptosis in cardiac myocytes: the role of inducible nitric oxide synthase induction and peroxynitrite generation. Circ. Res. 85:829-840.

57. Takuma, K., et al. 2001. Anti-apoptotic effect of cGMP in cultured astrocytes: inhibition by cGMPdependent protein kinase of mitochondrial permeable transition pore. J. Biol. Chem. 276:48093-48099.

58. Miyata, A., Kangawa, K., and Matsuo, H. 1986. Molecular forms of atrial natriuretic peptides in rat tissues and plasma. J. Hypertens. 4:S9-S11.

59. Murthy, K.K., Thibault, G., and Cantin, M. 1988. Contribution of blood and systemic circulation to the processing of pro-(atrial natriuretic factor). Biochem. J. 250:665-670.

60. Kuhn, M., et al. 2004. Left ventricular assist device support reverses altered cardiac expression and function of natriuretic peptides and receptors in end-stage heart failure. Cardiovasc. Res. 64:308-314.

61. Feil, R., Lohmann, S.M., de Jonge, H., Walter, U., and Hofmann, F. 2003. Cyclic GMP-dependent protein kinases and the cardiovascular system: insights from genetically modified mice [review].
Circ. Res. 93:907-916.

62. Tsutamoto, T., et al. 1993. Possibility of downregulation of atrial natriuretic peptide receptor coupled to guanylate cyclase in peripheral vascular beds of patients with chronic severe heart failure. Circulation. 87:70-75

63. Shevchuk, N.A., et al. 2004. Construction of long DNA molecules using long PCR-based fusion of several fragments simultaneously. Nucleic Acids Res. 32:e19.

64. Melendez, J., et al. 2002. Activation of pyk2/related focal adhesion tyrosine kinase and focal adhesion kinase in cardiac remodeling. J. Biol. Chem. 277:45203-45210.

65. Sussman, M.A., et al. 1999. Pathogenesis of dilated cardiomyopathy: molecular, structural, and population analyses in tropomodulin-overexpressing transgenic mice. Am. J. Pathol. 155:2101-2113.

66. Melendez, J., et al. 2004. Cardiomyocyte apoptosis triggered by RAFTK/pyk2 via Src kinase is antagonized by paxillin. J. Biol. Chem. 279:53516-53523.

67. Morrison, L.E., Whittaker, R.J., Klepper, R.E., Wawrousek, E.F., and Glembotski, C.C. 2004. Roles for alphaB-crystallin and HSBP2 in protecting the myocardium from ischemia-reperfusion-induced damage in a KO mouse model. Am. J. Physiol. Heart Circ. Physiol. 286:H847-H855. 